\title{
3D finite element formulation for mechanical-electrophysiological coupling in axonopathy
}

\author{
Man Ting Kwong, ${ }^{a, *}$, Fabio Bianchi ${ }^{a}$, Majid Malboubi ${ }^{a}$, Julián Andrés \\ García-Grajales $^{\mathrm{a}, \mathrm{c}}$, Lina Homsi ${ }^{\mathrm{b}}$, Mark Thompson ${ }^{\mathrm{a}}$, Hua $\mathrm{Ye}^{\mathrm{a}}$, Ludovic \\ Noels $^{\mathrm{b}}$, Antoine Jérusalem ${ }^{\mathrm{a}, *}$ \\ ${ }^{a}$ Department of Engineering Science, Parks Road, University of Oxford, UK, OX1 3PJ \\ ${ }^{b}$ Aerospace and Mechanical Engineering Department, University of Liège, Liège, Belgium \\ ${ }^{c}$ Mathematical Institute, University of Oxford, Oxford, UK, OX2 6GG
}

\begin{abstract}
Traumatic injuries to the central nervous system (brain and spinal cord) have recently been put under the spotlight because of their devastating socioeconomical cost. At the cellular scale, recent research efforts have focussed on primary injuries by making use of models aimed at simulating mechanical deformation induced axonal electrophysiological functional deficits. The overwhelming majority of these models only consider axonal stretching as a loading mode, while other modes of deformation such as crushing or mixed modes - highly relevant in spinal cord injury - are left unmodelled. To this end, we propose here a novel 3D finite element framework coupling mechanics and electrophysiology by considering the electrophysiological HodgkinHuxley and Cable Theory models as surface boundary conditions introduced directly in the weak form, hence eliminating the need to geometrically account for the membrane in its electrophysiological contribution. After validation against numerical and experimental results, the approach is leveraged to model an idealised axonal dislocation injury. The results show that the sole consideration of induced longitudinal stretch following transverse loading of a node of Ranvier is not necessarily enough to capture the extent of axonal electrophysiological deficit and that the non-axisymmetric loading of the node participates to a larger extent to the subsequent damage. On the contrary,
\end{abstract}

\footnotetext{
${ }^{*}$ Corresponding authors

Email addresses: man.kwong@eng.ox.ac.uk (Man Ting Kwong ), antoine.jerusalem@eng.ox.ac.uk (Antoine Jérusalem )
} 
a similar transverse loading of internodal regions was not shown to significantly worsen with the additional consideration of the non-axisymmetric loading mode.

Keywords: mechanical-electrophysiological coupling, finite element method, neuronal membrane, axonal injury, Hodgkin-Huxley, Cable Theory

\section{Introduction}

In traumatic brain injuries (TBIs) and spinal cord injuries (SCIs), the central nervous system is subjected to multiple mechanical loading modes, e.g., stretch, compression, shear or a combination of those $[1,2,3]$. The mechanical disturbances compromise the structural integrity of the tissue and underlying cells, in turn inducing electrophysiological alterations at various scales $[4,5,6,7,8,9,10,11,12]$.

At the subcellular scale, the dynamics of voltage gated sodium $\left(N a_{V}\right)$ ion channels embedded in patches of membrane subjected to micropipette suction was observed to be slightly accelerated (the so-called "left shift") [10]. At the cellular scale, an increase in action potential (AP) amplitude and a shorter refractory period were observed in an in vivo mouse model of mild TBI leading to axonal swelling [8]. Similarly, acute compression applied to guinea pig spinal cord white matter resulted into a reduction in compound action potential (CAP), occuring concurrently with paranodal myelin damage and membrane disruption $[6,9,7]$. At the cellular network scale, acute stretch injuries were seen to decrease AP firing and network bursting activity in cultured rat neocortical neurons under high strain rate loading [4, 5]. Similarly, a TBI inducing blast on an in vitro network of hippocampal neurons was observed to compromise its firing synchronisation $[13,14]$.

Computational studies explicitly modelling electrophysiological alterations are often used to rationalise experimentally observed damage mechanisms $[15,16,17]$. Babbs and Shi $[15]$ used increasing node width to computationally simulate mild retraction of myelin caused by stretch and crush injuries, while more severe retraction and detachment of paranodal myelin were generalised by decreasing paranodal resistance in their simulation. Boucher et al. [16] modelled the trauma induced coupled left shift dynamics of $N a_{V} 1.6$ channels observed experimentally by Wang et al. [10] by displacing the membrane potential towards a more hyperpolarised state. Volman and Ng [17] 
proposed a compartmentalised axon model to consider separately nodes of Ranvier, paranodes and juxtaparanodes, and focussed their investigation on the electrophysiological alteration caused by the nodal junction demyelination observed experimentally [9]. While the studies mentioned above all capture electrophysiological alterations arising from geometrical alterations, they fall short of directly relating the electrophysiological alterations to mechanical deformation (i.e., any mechanical deformation automatically affects the electrophysiolgical model), hence limiting the ability to model graded damage under varying degrees of deformation with one unique model $[18,10,7]$.

To this end, Jérusalem et al. [19] proposed a 1D finite difference model to capture the longitudinal strain and strain rate dependence of electrophysiological alteration by relating the $N a_{V}$ and voltage gated potassium $\left(K_{V}\right)$ ion channels dynamics to the strain in the membrane. This model aimed at capturing the recovery of CAP amplitude up to 30 minutes post white matter stretch in experiments conducted by Shi and Whitebone [7]. Other formulations have been since proposed [20, 21]. Because of their axisymmetric assumptions, they suffer equally from the same limitations in loading modes as the earlier reference.

Recent work by Cinelli et al. [22] proposed the use of electro-thermal equivalences and piezoelectric effect to couple mechanics and electrophysiology in nerves. Their finite element (FE) model was implemented on the commercial software ABAQUS [23]. Their model considers the extracellular matrix, membrane and intracellular matrix as separate element types. The strain based electrophysiology damage model proposed by Jérusalem et al. [19] was adopted in this model but the ion channel dynamics damage was not directly linked to the deformation. While the 3D FE approach allows for multiple loading modes, the required spatial discretisation of the axonal membrane (approximately a thousandth of the axon diameter) for a fully geometrically conserving model remains computationally expensive.

In this paper, we propose a scalable FE framework aimed at modelling axonal electrophysiological alteration directly induced by 3D non-axisymmetric mechanical deformations. To this end, an additional degree of freedom, electrical potential, is considered at the nodes. Poisson's equation is used as the governing potential equation while the AP propagation at the internodal and nodal membrane is captured by the Cable Theory (CT) and Hodgkin Huxley (HH) models, respectively, as electro-mechanically coupled boundary conditions introduced directly in the weak form. The advantage of this approach is its ability to incorporate these phenomenological electrophysiolo- 
gical equations into a 3D framework without the explicit inclusion of extra 3D membrane elements. Details of the governing equations and the FE formulation are presented in Section 2. The model, implemented in the open source FE platform Gmsh [24, 25], is validated in Section 3. Finally, Section 4 illustrates the flexibility of the method with an idealised study of axonal indentation and highlights to consider 3D deformation (as opposed to solely 1D) to successfully model axonal injury.

\section{Finite element framework}

\subsection{Mechanics}

The balance of linear momentum for a material point of coordinate $\mathbf{X}$ in the reference configuration $\Omega_{0}$ and of coordinate $\mathbf{x}$ in the current configuration $\Omega$ reads:

$$
\operatorname{Div} \mathbf{P}(\mathbf{X})+\rho_{0} \mathbf{b}=\rho_{0} \ddot{\mathbf{X}}(\mathbf{X})
$$

where $\mathbf{P}, \rho_{o}$ and $\mathbf{b}$ are the first Piola-Kirchhoff stress, the material density and the body force in the reference configuration, respectively. "Div" is the divergence operator formulated in the reference configuration. In indicial notation, Equation 1 reads:

$$
P_{i J, J}+\rho_{0} b_{i}=\rho_{0} \ddot{x}_{i}
$$

Neumann and Dirichlet boundary conditions on the corresponding boundaries $\partial \Omega_{0}^{n}$ and $\partial \Omega_{0}^{d}$ can be formulated as

$$
\left\{\begin{aligned}
\mathbf{P} \cdot \mathbf{N}=\overline{\mathbf{T}}, & \forall \mathbf{X} \in \partial \Omega_{0}^{n} \\
\mathbf{u}=\overline{\mathbf{u}}, & \forall \mathbf{X} \in \partial \Omega_{0}^{d}
\end{aligned}\right.
$$

where $\mathbf{u}=\mathbf{x}-\mathbf{X}$ is the material point displacement, $\mathbf{N}$ is the boundary normal, and $\overline{\mathbf{T}}$ and $\overline{\mathbf{u}}$ are the imposed traction and displacement on their respective boundaries in the reference configuration.

The weak form of the mechanical part then reads: for all admissible virtual displacements $\boldsymbol{\eta}$,

$$
a_{\mathbf{u u}}(\mathbf{u}, \boldsymbol{\eta})=l_{\mathbf{u}}(\boldsymbol{\eta})
$$

where

$$
\left\{\begin{array}{l}
a_{\mathbf{u u}}(\mathbf{u}, \boldsymbol{\eta})=\iiint_{\Omega_{0}} P_{i J}(\mathbf{u}) \eta_{i, J} d V_{0} \\
l_{\mathbf{u}}(\boldsymbol{\eta})=\iiint_{\Omega_{0}} \rho_{0} b_{i} \eta_{i} d V_{0}+\iint_{\partial \Omega_{0}^{n}} \bar{T}_{i} \eta_{i} d S_{0}
\end{array}\right.
$$


Note that, without loss of generality, the dynamic term is dropped here and subsequently.

The final FE problem for the mechanical part is defined by:

$$
\mathbf{F}_{\mathbf{u}}^{e x t}=\mathbf{F}_{\mathbf{u}}^{i n t}
$$

where the mechanical external and internal force vectors $\mathbf{F}_{\mathbf{u}}^{\text {ext }}$ and $\mathbf{F}_{\mathbf{u}}^{\text {int }}$ are given by:

$$
\left\{\begin{aligned}
F_{\mathbf{u}, i a}^{\text {ext }} & =\iiint_{\Omega_{0}} \rho_{0} b_{i} \mathcal{N}_{a} d V_{0}+\iint_{\partial \Omega_{0}^{n}} \bar{T}_{i} \mathcal{N}_{a} d S_{0} \\
F_{\mathbf{u}, i a}^{i n t} & =\iiint_{\Omega_{0}} P_{i J}\left(\mathbf{u}_{\mathbf{h}}\right) \mathcal{N}_{a, J} d V_{0}
\end{aligned}\right.
$$

where the FE displacement vector $\mathbf{u}_{h}$ is estimated from the nodal displacement vector $\mathbf{u}_{a}$ by use of the shape functions $\mathcal{N}_{a}$ :

$$
\mathbf{u}_{h}=\Sigma_{a} \mathcal{N}_{a}(\mathbf{X}) \mathbf{u}_{a}
$$

\subsection{Electrophysiology}

The potential $V(\mathbf{x})$ in a material of constant resistivity $\rho_{c}$ (is taken here as the cytoplasm resistivity), with a current source density $\tilde{\rho}$, is described at all material points of the body $B$ in the current configuration by the Poisson equation:

$$
\Delta V=-\tilde{\rho} \rho_{c}
$$

where " $\Delta$ " is the Laplace operator. While $\tilde{\rho}$ may be essential in some cases, e.g., when considering secondary injury mechanisms associated with mitochondrial calcium transport disruption [26, 27], it is neglected here as a first approximation and Equation (9) is reduced to Laplace's equation $(\Delta V=0)$.

At the boundary of the deformed body $B$, the boundary conditions are assumed to follow either the $\mathrm{CT}$ or $\mathrm{HH}$ model, depending on whether the region of interest is an internodal region's boundary $\left(\partial \Omega^{C T}\right)$ or a node of Ranvier's boundary $\left(\partial \Omega^{H H}\right)$, see Figure 1 . While the former is enveloped by successive layers of myelin, the latter is an active membrane with $N a_{V}$ and $K_{V}$ ion channels given free access to the extracellular medium.

The current $i_{n}$ flowing out of a patch of membrane surface $d S$ with a potential $V$ follows the relation:

$$
i_{n}=-\frac{d S}{\rho_{c}} \nabla_{\mathbf{n}} V, \forall \mathbf{x} \in \partial \Omega^{C T} \cup \partial \Omega^{H H}
$$



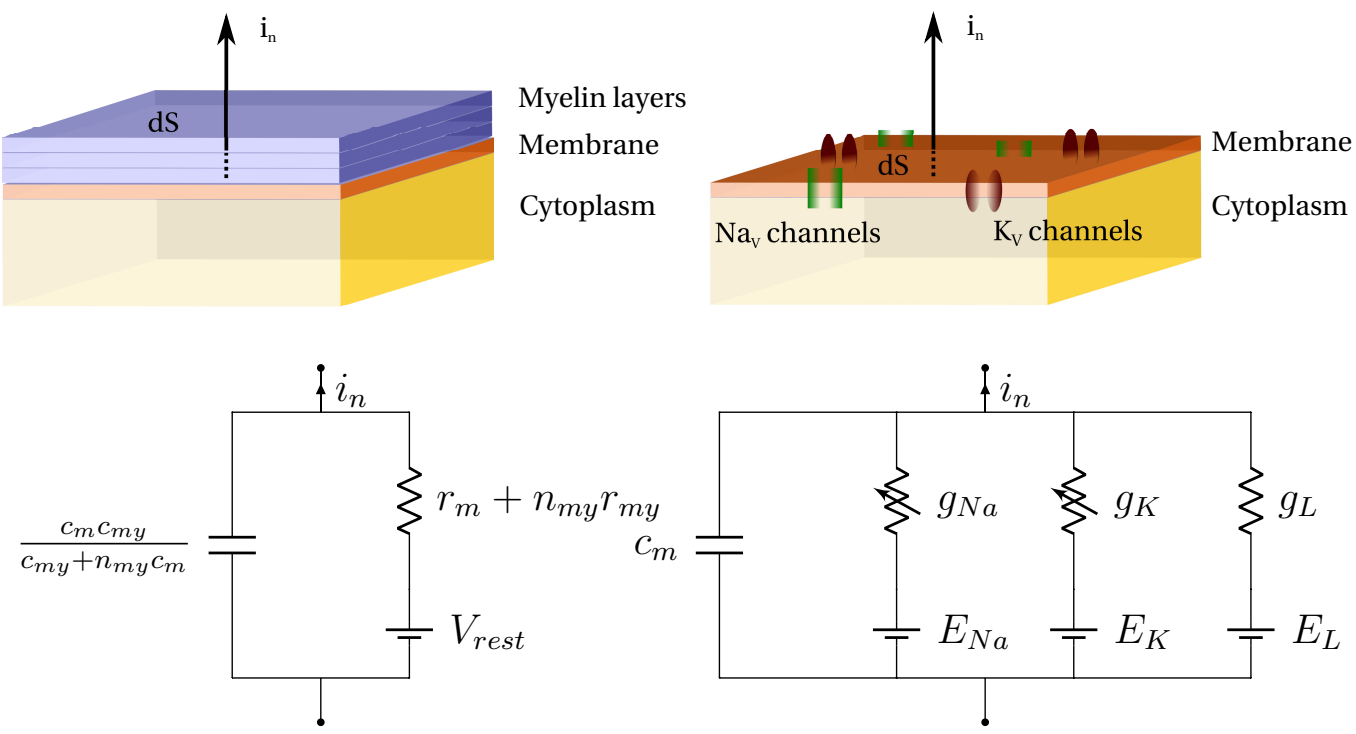

(a) Internode

(b) Node

Figure 1: Axonal membrane outward current flux, a) either impeded by myelin layers in the internodal regions, b) or governed by the dynamics of the gating of the $N a_{V}$ and $K_{V}$ channels in the nodes of Ranvier, modelled by the CT and HH models, respectively.

where $\mathbf{n}$ is the normal to the membrane (pointing away from the cell) in the current configuration.

The identification of $i_{n}$ in both models (see CT and HH models in Figure 1) for a patch of membrane of area $d S$ leads to:

$$
\begin{cases}i_{n}=\frac{c_{m} c_{m y}}{c_{m y}+n_{m y} c_{m}} \frac{\partial V}{\partial t}+\frac{1}{r_{m}+n_{m y} r_{m y}}\left(V-V_{\text {rest }}\right), & \forall \mathbf{x} \in \partial \Omega^{C T} \\ i_{n}=c_{m} \frac{\partial V}{\partial t}+g_{N a}\left(V-E_{N a}\right)+g_{K}\left(V-E_{K}\right)+g_{L}\left(V-E_{L}\right), & \forall \mathbf{x} \in \partial \Omega^{H H}\end{cases}
$$

where $r_{m}$ and $c_{m}$ are the membrane resistance and capacitance, $r_{m y}$ and $c_{m y}$ are the individual myelin layer's resistance and capacitance for $n_{m y}$ number of layers. $V_{\text {rest }}$ is the resting potential. $g_{N a}, g_{K}$ and $g_{L}$ are the $N a_{V}$ and $K_{V}$ ion channels, and leak conductances; $E_{N a}$ and $E_{K}$ are their reversal potentials. Their relationship with the specific membrane and myelin layer resistivities, $\rho_{m}$ and $\rho_{m y}$, membrane and myelin layer equivalent electric constants, $C_{m}$ and $C_{m y}$, and dynamic conductances $G_{N a}\left(V, \varepsilon_{s}\right), G_{K}\left(V, \varepsilon_{s}\right)$ (which can $a$ 
priori be dependent of the surface strain $\varepsilon_{s}$ and $G_{L}$, are listed below:

$$
\left\{\begin{array}{l}
g_{N a}=\frac{d S G_{N a}}{h_{m}}, g_{K}=\frac{d S G_{K}}{h_{m}}, g_{L}=\frac{d S G_{L}}{h_{m}} \\
r_{m}=\frac{\rho_{m} h_{m}}{d S}, r_{m y}=\frac{\rho_{m y} h_{m y}}{d S} \\
c_{m}=\frac{d S C_{m}}{h_{m}}, c_{m y}=\frac{d S C_{m y}}{h_{m y}}
\end{array}\right.
$$

where $h_{m}$ and $h_{m y}$ are the membrane and myelin layer thicknesses. Here the channel conductances are expressed with respect to the surface area in the current configuration, $d S$, however, $g_{N a}$ and $g_{K}$ can also be calculated with respect to $d S_{0}$, which will later be used to consider a patch of active membrane with a conserved number of ion channels.

Coupling Equations (10), (11) and (12):

$$
\left\{\begin{array}{rr}
\nabla_{\mathbf{n}} V=-\rho_{c}\left(\frac{C_{m} C_{m y}}{h_{m} C_{m y}+n_{m y} h_{m y} C_{m}} \frac{\partial V}{\partial t}+\frac{1}{h_{m} \rho_{m}+n_{m y} h_{m y} \rho_{m y}}\left(V-V_{\text {rest }}\right)\right), \\
\forall \mathbf{x} \in \partial \Omega^{C T} \\
\nabla_{\mathbf{n}} V=\frac{-\rho_{c}}{h_{m}}\left(C_{m} \frac{\partial V}{\partial t}+G_{N a}\left(V-E_{N a}\right)+G_{K}\left(V-E_{K}\right)+G_{L}\left(V-E_{L}\right)\right), \\
\forall \mathbf{x} \in \partial \Omega^{H H}
\end{array}\right.
$$

which is subsequently simplified to:

$$
\begin{cases}\nabla_{\mathbf{n}} V=f_{C T}\left(V, \frac{\partial V}{\partial t}, \varepsilon_{s}\right), & \forall \mathbf{x} \in \partial \Omega^{C T} \\ \nabla_{\mathbf{n}} V=f_{H H}\left(V, \frac{\partial V}{\partial t}, \varepsilon_{s}\right), & \forall \mathbf{x} \in \partial \Omega^{H H}\end{cases}
$$

$E_{L}$ is chosen such that $V=V_{\text {rest }}$ at rest $[19,28]$, i.e.,

$$
E_{L}=\left(1+\frac{G_{N a}\left(V_{\text {rest }}\right)+G_{K}\left(V_{\text {rest }}\right)}{G_{L}}\right) V_{\text {rest }}-\frac{\left(G_{N a}\left(V_{\text {rest }}\right) E_{N a}+G_{K}\left(V_{\text {rest }}\right) E_{K}\right)}{G_{L}}
$$

The weak form of the electrophysiological part then reads: for all admissible virtual potential $H$,

$$
a_{V V}(V, H)=l_{V}(H)
$$


where

$$
\left\{\begin{array}{l}
a_{V V}(V, H)=\iiint_{\Omega} V_{, i} H_{, i} d V \\
l_{V}(H, V, \mathbf{u})=\iiint_{\Omega} \tilde{\rho} \rho_{c} H d V+\iint_{\partial \Omega^{C T}} H f_{C T}(V, \mathbf{u}) d S+\iint_{\partial \Omega^{H} H} H f_{H H}(V, \mathbf{u}) d S
\end{array}\right.
$$

Making use of Nanson's formula, $d S \mathbf{n}=J d S_{0} \mathbf{F}^{-T} \cdot \mathbf{N}$, where $\mathbf{F}=\frac{\partial \mathbf{x}}{\partial \mathbf{x}}$ is the deformation gradient tensor, Equation (17) can be rewritten in the reference configuration:

$$
\left\{\begin{aligned}
a_{V V}(V, H) & =\iiint_{\Omega_{0}} V_{, J} F_{J i}^{-1} H_{, K} F_{K i}^{-1} J d V_{0} \\
l_{V}(H, V, \mathbf{u}) & =\iiint_{\Omega_{0}} \tilde{\rho} \rho_{c} H J d V_{0} \\
& +\iint_{\partial \Omega_{0}^{C T}} H f_{C T}(V, \mathbf{u}) J N_{I} F_{I i}^{-1} n_{i} d S_{0} \\
& +\iint_{\partial \Omega_{0}^{H H}} H f_{H H}(V, \mathbf{u}) J N_{I} F_{I i}^{-1} n_{i} d S_{0}
\end{aligned}\right.
$$

where $J=\operatorname{det}(\mathbf{F})$ is the Jacobian and the subscript " 0 " refers to the reference configuration. Note that here $a_{V V}$ also indirectly depends on $\mathbf{u}$ through $\mathbf{F}$.

The final FE problem for the electrophysiological part is defined by:

$$
\mathbf{F}_{V}^{e x t}=\mathbf{F}_{V}^{i n t}
$$

where the electrophysiological external and internal force vectors $\mathbf{F}_{V}^{e x t}$ and $\mathbf{F}_{V}^{i n t}$ are given by:

$$
\left\{\begin{aligned}
F_{V, a}^{e x t}=l_{V}\left(\mathcal{N}_{a}, V_{h}, \mathbf{u}_{\mathbf{h}}\right)= & \iiint_{\Omega_{0}} \tilde{\rho} \rho_{c} \mathcal{N}_{a} J d V_{0} \\
& +\iint_{\partial \Omega_{0}^{C T}} \mathcal{N}_{a} f_{C T}\left(V_{h}, \mathbf{u}_{\mathbf{h}}\right) J \mathcal{N}_{I} F_{I i}^{-1} n_{i} d S_{0} \\
& +\iint_{\partial \Omega_{0}} \mathcal{N}_{a} f_{H H}\left(V_{h}, \mathbf{u}_{\mathbf{h}}\right) J \mathcal{N}_{I} F_{I i}^{-1} n_{i} d S_{0} \\
F_{V_{h}, a}^{i n t}=a_{V V}\left(V_{h}, \mathcal{N}_{a}\right)= & \iiint_{\Omega_{0}} V_{h, J} F_{J i}^{-1} \mathcal{N}_{a, K} F_{K i}^{-1} J d V_{0}
\end{aligned}\right.
$$

where the FE potential vector $V_{h}$ is estimated from the nodal potential vector $V_{a}$ by use of the shape functions $\mathcal{N}_{a}$ :

$$
V_{h}=\Sigma_{a} \mathcal{N}_{a}(\mathbf{X}) V_{a}
$$

Note that the same shape functions were used for both discretisations. 


\subsection{Coupled problem}

The coupled FE problem consists in solving:

$$
\mathbf{F}^{e x t}=\mathbf{F}^{\text {int }}
$$

where the coupled external and internal force vectors $\mathbf{F}^{\text {ext }}$ and $\mathbf{F}^{\text {int }}$ are assembled from their physics counterparts:

$$
\mathbf{F}^{e x t}=\left(\begin{array}{c}
\mathbf{F}_{\mathbf{u}}^{e x t} \\
\mathbf{F}_{V}^{e x t}
\end{array}\right), \mathbf{F}^{i n t}=\left(\begin{array}{c}
\mathbf{F}_{\mathbf{u}}^{i n t} \\
\mathbf{F}_{V}^{i n t}
\end{array}\right)
$$

In a non-linear implicit problem, the residual $\mathbf{r}=\mathbf{F}^{\text {int }}-\mathbf{F}^{\text {ext }}$ is iteratively decreased to zero (or close enough) through the Newton-Raphson method. To this end, the stiffness matrix is defined as the derivative of the residual:

$$
\mathbf{K}=\left(\begin{array}{ll}
\mathbf{K}^{\mathbf{u u}} & \mathbf{K}^{\mathbf{u} V} \\
\mathbf{K}^{V \mathbf{u}} & \mathbf{K}^{V V}
\end{array}\right)=\left(\begin{array}{ll}
\frac{\partial \mathbf{r}_{\mathbf{u}}}{\partial \mathbf{u}} & \frac{\partial \mathbf{r}_{\mathbf{u}}}{\partial V} \\
\frac{\partial \mathbf{r}_{V}}{\partial \mathbf{u}} & \frac{\partial \mathbf{r}_{V}}{\partial V}
\end{array}\right)
$$

By solving both CT and HH equations using the forward difference scheme (see Appendix A.1 and Appendix A.2), the stiffness matrix terms can be written as:

$$
\begin{gathered}
K_{i a k b}^{\mathbf{u u}}=\iiint_{\Omega_{0}} \frac{\partial P_{i J}}{\partial F_{k L}} \mathcal{N}_{a, J} \mathcal{N}_{b, L} d V_{0} \\
\mathbf{K}^{\mathbf{u} V}=\mathbf{0} \\
K_{a b}^{V V}=\iiint_{\Omega_{0}} \mathcal{N}_{b, J} F_{J i}^{-1} \mathcal{N}_{a, K} F_{K i}^{-1} J d V_{0} \\
+\iint_{\partial \Omega_{0}^{C T}} \mathcal{N}_{a} \frac{\rho_{c} C_{m} C_{m y}}{\left(h_{m} C_{m y}+n_{m y} h_{m y} C_{m}\right) \Delta t} \mathcal{N}_{b} J N_{I} F_{I i}^{-1} n_{i} d S_{0} \\
+\iint_{\partial \Omega_{0}^{H H}} \mathcal{N}_{a} \frac{\rho_{c} C_{m}}{h_{m} \Delta t} \mathcal{N}_{b} J N_{I} F_{I i}^{-1} n_{i} d S_{0}
\end{gathered}
$$




$$
\begin{aligned}
K_{a k b}^{V u}=\frac{\partial r_{a}^{V}}{\partial u_{k b}}= & \iiint_{\Omega_{0}} V_{c} \mathcal{N}_{c, J} \frac{\partial F_{J i}^{-1}}{\partial u_{k b}} \mathcal{N}_{a, K} F_{K i}^{-1} J d V_{0} \\
& +\iiint_{\Omega_{0}} V_{c} \mathcal{N}_{c, J} F_{J i}^{-1} \mathcal{N}_{a, K} \frac{\partial F_{K i}^{-1}}{\partial u_{k b}} J d V_{0} \\
& +\iiint_{\Omega_{0}} V_{c} \mathcal{N}_{c, J} F_{J i}^{-1} \mathcal{N}_{a, K} F_{K i}^{-1} \frac{\partial J}{\partial u_{k b}} d V_{0} \\
& -\iiint_{\Omega_{0}} \tilde{\rho} \rho_{c} \mathcal{N}_{a} \frac{\partial J}{\partial u_{k b}} d V_{0} \\
& -\iint_{\partial \Omega_{0}^{C T}} \mathcal{N}_{a} \frac{\partial f_{C T}}{\partial u_{k b}} J N_{I} F_{I i}^{-1} n_{i} d S_{0} \\
& -\iint_{\partial \Omega_{0}^{C T}} \mathcal{N}_{a} f_{C T} N_{I} \frac{\partial\left(J F_{I i}^{-1} n_{i}\right)}{\partial u_{k b}} d S_{0} \\
& -\iint_{\partial \Omega_{0}^{H H}} \mathcal{N}_{a} \frac{\partial f_{H H}}{\partial u_{k b}} J N_{I} F_{I i}^{-1} n_{i} d S_{0} \\
& -\iint_{\partial \Omega_{0}^{H H}} \mathcal{N}_{a} f_{H H} N_{I} \frac{\partial\left(J F_{I i}^{-1} n_{i}\right)}{\partial u_{k b}} d S_{0}
\end{aligned}
$$

where

$$
\frac{\partial\left(J F_{I i}^{-1} n_{i}\right)}{\partial u_{k b}}=J F_{J k}^{-1} \mathcal{N}_{b, J} F_{I i}^{-1} n_{i}-J F_{I k}^{-1} \mathcal{N}_{b, K} F_{K i}^{-1} n_{i}+J F_{I i}^{-1} \frac{\partial n_{i}}{\partial u_{k b}}
$$

The terms in $\frac{\partial f_{C T}}{\partial u_{k b}}$ and $\frac{\partial f_{H H}}{\partial u_{k b}}$ were found to be negligible, but their derivations are provided along with $\frac{\partial n_{i}}{\partial u_{k b}}$ in Appendix A.3, Appendix A.4 and Appendix A.5. Note finally that all integrals (both in volume and on surface) are numerically calculated using Gauss quadrature.

\subsection{Electrophysiological validation}

The aforementioned 3D FE framework was implemented in the open source FE code Gmsh [24, 25]. In order to validate the electrophysiological 
implementation of the $\mathrm{CT}$ and $\mathrm{HH}$ boundary conditions, both were modelled separately and, in the absence of deformation, validated against the results of two other numerical codes: Neurite, a 1D finite difference framework for mechanical electrophysiological coupling [19, 28], and a Matlab 1D FE code with CT and $\mathrm{HH}$ applied as boundary conditions in a similar fashion, and solved using the Newton-Raphson iterative scheme, see Appendix B for the full study and Appendix $\mathrm{C}$ for the Matlab 1D code.

\section{Model validation}

The model was validated by studying the electrophysiological alteration of neurons during stretching. Mechanical stretching was applied to a flexible substrate, on which excitable cells were cultured. Voltage clamp was used to measure the electrophysiology of both control and stretched cells. In the following, the experimental setup and the numerical simulations are presented.

\subsection{Experimental materials and methods}

\subsubsection{Cell culture}

Rat F11 cells (ATCC, UK), an immortalised hybrid of rat dorsal root ganglion (DRG) neurons and neuroblastoma cells, were chosen due to their uni-

que combination of fast proliferation and spontaneous action potential firing $[29,30]$. Cells were cultured in high-glucose Dulbeccos modified eagle medium (DMEM, ThermoFisher, UK), supplemented with $1 \%$ penicillin/streptomycin (P/S, Sigma Aldrich, UK) and $10 \%$ foetal bovine serum (FBS, ThermoFisher, UK). After expansion, cells were resuspended and seeded on deformable substrates at a density of 50 cells $/ \mathrm{mm}^{2}$, to allow further expansion during differentiation. F11s were differentiated in high-glucose DMEM (ThermoFisher, UK), supplemented with $1 \%$ FBS, $1 \%$ P/S, $0.5 \%$ Insulin Transferrin Selenium (ITS, ThermoFisher, UK), 10 mol 3-Isobutyl-1-methylxanthine (IBMX, Sigma Aldrich, UK), $50 \mathrm{ng} / \mathrm{ml}$ Nerve Growth Factor (NGF, Peprotech, UK), $2 \mu \mathrm{mol}$ Retinoic Acid (Sigma Aldrich, UK) and $0.5 \mathrm{mmol}$ Bromoadenosine 3,5-cyclic monophosphate. Cells were differentiated for 5 days prior to stretch experiments.

\subsubsection{Cell stretching}

Cells were stretched by deformation of underlying culture substrate using a custom-built uniaxial stretching device [31]. This device allows simultaneous displacement-controlled cell stretching and single cell electrophysiology 
by patch clamping. Cell populations on deformable substrates were stretched to $45 \%$ substrate strain, and ion currents measured.

\subsubsection{Electrophysiology}

The experimental setup for electrophysiological recording consisted of a Digidata 1440 A Digitizer and a MultiClamp 700B Amplifier piloted through pCLAMP 10 Software (all from Molecular Devices, CA). Glass micropipettes were pulled from thin wall borosilicate capillary tubes (BF100-78-10, Sutter Instruments, CA), using a Flaming/Brown micropipette puller (Model P1000, Sutter Instruments, CA), to a final resistance of 8 - $15 \mathrm{M} \Omega$. Pulling parameters were optimised according to previous work [32] in order to obtain the desired shape and surface properties of micropipettes. The intracellular solution contained: $140 \mathrm{mmol} \mathrm{KCl}, 5 \mathrm{mmol} \mathrm{NaCl}, 0.5 \mathrm{mmol} \mathrm{CaCl}_{2}, 2 \mathrm{mmol}$ $\mathrm{MgCl}_{2}, 10 \mathrm{mmol}$ HEPES, $1 \mathrm{mmol}$ GTP, $2 \mathrm{mmol}$ ATP, with $\mathrm{pH}$ adjusted to 7.4 by addition of $\mathrm{KOH}$ and osmolarity adjusted to $300 \mathrm{mOsml}^{-1}$ by glucose addition. The bath solution contained: $130 \mathrm{mmol} \mathrm{NaCl}, 5 \mathrm{mmol} \mathrm{KCl,} 2 \mathrm{mmol}$ $\mathrm{CaCl}_{2}, 1 \mathrm{mmol} \mathrm{MgCl}_{2}, 10 \mathrm{mmol}$ glucose, $10 \mathrm{mmol}$ HEPES, with $\mathrm{pH}$ adjusted to 7.4 by addition of $\mathrm{NaOH}$ and osmolarity adjusted to $300 \mathrm{mOsml}^{-1}$ by glucose addition. To evoke voltage dependent currents, cells were stimulated with depolarising pulses at $0 \mathrm{mV}$ and currents were recorded in voltage clamp mode. Cells were chosen for patch clamping based on morphology, with selected cells displaying at least three processes and distinct pyramidal neuron-like somatas. Following establishment of whole-cell patches, only cells displaying both inwards and outwards currents were used for data analysis. Cells were patched on deformable membranes before stretch (control), and during an applied whole cell strain of $45 \%$. Voltage clamp traces were analysed in Clampfit (Molecular Devices, CA). Traces were cropped to eliminate pipette capacitance artifacts, and average traces for stretched and control cells plotted at different voltage clamp levels.

\subsection{Numerical simulation}

\subsubsection{Geometry and discretisation}

In order to compare the numerical simulations to experimental voltage clamp measurement, the membrane in the neighbourhood of the voltage clamp was simulated. The cell within the micropipette was approximated by a frustum of a cone (with a height of $2 \mu \mathrm{m}$, and upper and lower radii 
of 0.75 and $0.5 \mu \mathrm{m}$, respectively) and the cytoplasm immediately underneath the pipette was represented by a $3 \times 1 \times 3 \mu \mathrm{m}^{3}$ rectangular parallelepiped, see Figure 2. The resulting geometry was discretised with 4,249 quadratic tetrahedral elements.

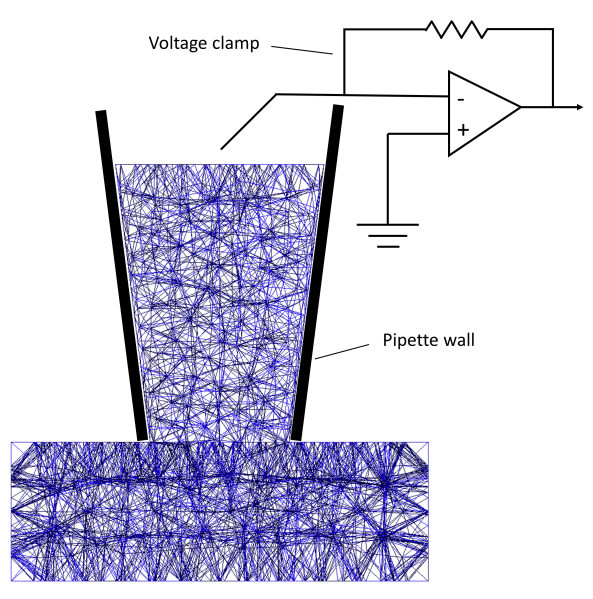

(a) Finite element mesh representing a rat F11 cell under voltage clamp.

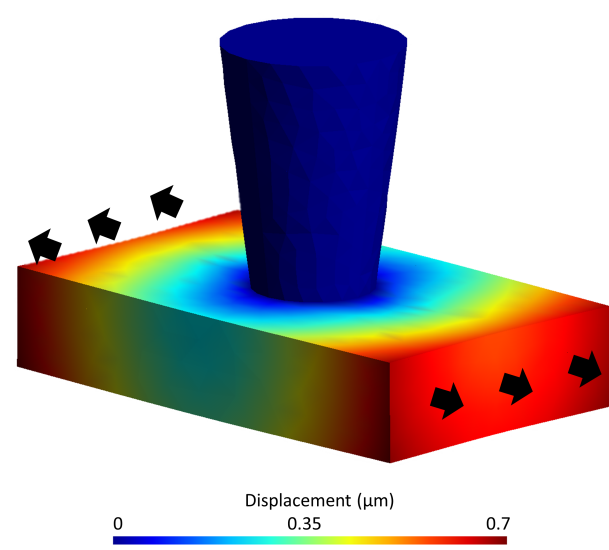

(b) Deformation of a rat F11 cell simulating substrate stretch.

Figure 2: Deformation of a rat F11 cell under substrate stretch simulated by pressure boundary conditions applied to the lateral sides of the cytoplasm domain.

\subsubsection{Material law and numerical solver}

As presented in Section 2.2, the Poisson's equation was chosen to govern the electrical charge distribution in the bulk of the cytoplasm, with $\rho_{c}$ being $1.87 \Omega \mathrm{m}$. The electrophysiological parameters are the same as in Ref. $[19,28]$, see Table 1. An explicit scheme was used for solving the electrophysiological ion channel dynamics variables of the $\mathrm{HH}$ boundary condition equations. The simulation time step was chosen based on the convergence of the electrophysiological equations. 


\begin{tabular}{lll}
\hline & Parameter & Value \\
\hline \hline$V_{\text {rest }}$ & Resting potential & $-65 \mathrm{mV}$ \\
$\rho_{c}$ & Cytoplasm resistivity & $1.87 \Omega \mathrm{m}$ \\
$\rho_{m}$ & Membrane resistivity & $2.5 \times 10^{6} \Omega \mathrm{m}$ \\
$\rho_{m y}$ & Myelin layer resistivity & $4.44 \times 10^{6} \Omega \mathrm{m}$ \\
$C_{m}$ & Membrane capacitance & $4 \times 10^{-11} \mathrm{~F} \mathrm{~m}^{-1}$ \\
$C_{m y}$ & Myelin capacitance & $1.8 \times 10^{-10} \mathrm{~F} \mathrm{~m}^{-1}$ \\
$h_{m}$ & Membrane thickness & $4 \times 10^{-9} \mathrm{~m}$ \\
$h_{m y}$ & Myelin layer thickness & $1.08 \times 10^{-9} \mathrm{~m}$ \\
$n_{m y}$ & Number of myelin layers & 45 \\
$\bar{G}_{N a}$ & $N a_{V}$ Reference conductivity & $4.8 \times 10^{-6} \mathrm{~S} \mathrm{~m}^{-1}$ \\
$\bar{G}_{K}$ & $K_{V}$ Reference conductivity & $1.44 \times 10^{-6} \mathrm{~S} \mathrm{~m}^{-1}$ \\
$G_{L}$ & Leak conductivity & $1.2 \times 10^{-8} \mathrm{~S} \mathrm{~m}^{-1}$ \\
$E_{N a}^{0}$ & $N a_{V}$ Reference reversal potential & $49.5 \mathrm{mV}$ \\
$E_{K}^{0}$ & $K_{V}$ Reference reversal potential & $-77.5 \mathrm{mV}$ \\
\hline
\end{tabular}

Table 1: Electrophysiological parameter values.

Mechanical membrane creep during patch clamp experiments has been previously observed [18], however the time scale of the creep was more than an order of magnitude larger than the time scale of an AP. A linear elastic material law along with a linear static implicit scheme were therefore considered adequate as a first approximation for modelling the cell's mechanical behaviour. Note that future implementations where material irreversible deformation (e.g., plasticity) can directly be fed into the electrophysiological models as additional damage parameters are straightforward. The mechanical material properties are listed in Table 2.

\begin{tabular}{lll}
\hline & Parameter & Value \\
\hline \hline$E$ & Young's modulus & $165.920 \mathrm{kPa}$ \\
$\rho$ & Material density & $993 \mathrm{~kg} \mathrm{~m}^{-3}$ \\
$\nu$ & Poisson's ratio & 0.3 \\
\hline
\end{tabular}

Table 2: Mechanical material properties for axon cytoplasm.

\subsubsection{Boundary conditions}

Opposite Neumann boundary conditions of $3.1 \mathrm{kPa}$ leading to $45 \%$ strain were applied to two lateral sides of the cytoplasm domain to simulate the 
experimental stretch deformation. The remaining faces of the cytoplasm were allowed to deform in the lateral and vertical directions. The top face of the box representing the membrane outside of the pipette was free to deform with the cytoplasm except in the vertical direction. The boundaries within the pipette were assumed to be static and fixed in displacement. The stretch of the cell was assumed to remain constant during the electrophysiological measurement (no creep), therefore the pressure boundary conditions were constant during the simulated AP propagation. The rupture of the cell membrane within the pipette in the whole cell configuration was represented by imposing a voltage Dirichlet boundary condition of $0 \mathrm{mV}$ to the top face of the frustum. The boundary representing the membrane in contact with the pipette was set to have no electrical flux across, while the distal outer faces of the cytoplasm away from the pipette were free of current flow. Hodgkin-Huxley Neumann boundary conditions were considered for the top face of the box representing the membrane outside of the pipette. The relationship between electrophysiological change and geometrical deformation is not yet fully established experimentally, therefore three different scenarios were considered in the validation study:

1. No change. The model described above is used as such with $E_{N a}=E_{N a}^{0}$ and $E_{K}=E_{K}^{0}$, i.e., no damage is considered. In this case, as the area of the membrane is changing during deformation, the electrophysiological properties vary accordingly, i.e., the integrals in Equation (17) are done with respect to the deformed configuration. This model is in agreement with the fact that growing axons retain their electrophysiological properties per unit membrane area [33].

2. Number of ion channels is conserved. No irreversible damage is considered $\left(E_{N a}=E_{N a}^{0}\right.$ and $\left.E_{K}=E_{K}^{0}\right)$ but the ion channel activity reflects the fact that even stretched, the number of ion channels remains the same, i.e., the integrals related to the ion channels activity in Equations (17) need to be done with respect to the original configuration, see Equation (30).

3. Number of ion channel is conserved and they can be damaged. This model follows the approach proposed by Jérusalem et al. [19] to model the ion channel left shift related damage. In this model, Equation (30) is also used but the reversal potentials of the ion channels are modified to reflect such damage, see Equation (31). 


$$
\begin{aligned}
& \left\{\begin{aligned}
a_{V V}(V, H)= & \iiint_{\Omega} V_{, i} H_{, i} d V \\
l_{V}(H, V, \mathbf{u})= & \iiint_{\Omega} \tilde{\rho} \rho_{c} H d V+\iint_{\partial \Omega^{C T}} H f_{C T}(V, \mathbf{u}) d S \\
& +\iint_{\partial \Omega^{H H}} \frac{-\rho_{c}}{h_{m}}\left(C_{m} \frac{\partial V}{\partial t}+G_{L}\left(V-E_{L}\right)\right) d S \\
& +\iint_{\partial \Omega_{0}^{H H}} \frac{-\rho_{c}}{h_{m}}\left(G_{N a}\left(V-E_{N a}\right)+G_{K}\left(V-E_{K}\right)\right) d S_{0}
\end{aligned}\right. \\
& \left\{\begin{array}{ll}
E_{N a}=\alpha E_{N a}^{0} \\
E_{K}=\alpha E_{K}^{0}
\end{array} \text { where } \alpha= \begin{cases}1-\left(\frac{\varepsilon_{s}}{\tilde{\varepsilon}}\right)^{\gamma}, & \text { if } \varepsilon_{s}<\tilde{\varepsilon} \\
0, & \text { else. }\end{cases} \right.
\end{aligned}
$$

where $\gamma=2$ is a damage model exponent representing the sensitivity of the damage model to small vs. large deformation and $\tilde{\varepsilon} \in\{0.1,0.2,0.3\}$ is the threshold at which the membrane is considered fully damaged [19].

\subsection{Results and conclusions}

\subsubsection{Experimental results}

Figure 3 presents the mean normalised current of a patch under whole cell voltage clamp at $0 \mathrm{mV}$ for the control cells (blue line) and stretched cells (red line). Seven control cells and six stretched cells were measured, the respective error bars are presented as transparent bands. The experimental results were normalised by the maximum of the mean hyperpolarising current of the control cells. On average the control cells produced a more hyperpolarising current compare to the stretched cells.

\subsubsection{Simulation results}

The overall current flowing from the cell into the pipette was calculated by summing the vertical current flowing out of all the elements of the top face of the geometry. Figure 4 shows the normalised current profiles for the control cell with no mechanical deformation (blue line), stretched (blue dashed line), stretched with conserved number of ion channels (blue dotted line) and stretched with various membrane damage threshold ( $\tilde{\varepsilon}=0.1$ as solid red line, $\tilde{\varepsilon}=0.2$ as red dashed line, $\tilde{\varepsilon}=0.3$ as red dotted line). 


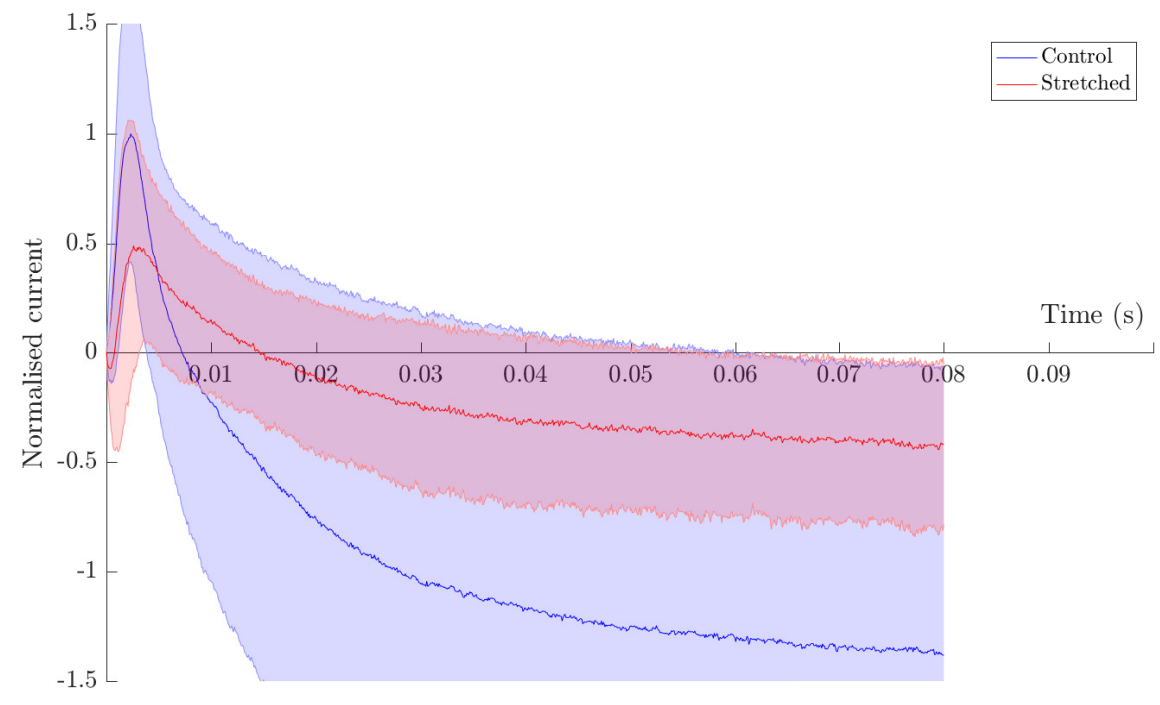

Figure 3: Normalised current from experimental whole cell voltage clamp at $0 \mathrm{mV}$ of control cell without stretch and cell at $45 \%$ stretch.

\subsubsection{Discussion}

In the whole cell voltage clamp configuration, the numerical model predicts that stretching the membrane will not lead to a significant alteration of the measured current when the model only considers geometrical deformation (Case 1) or considers the deformation with a conserved number of ion channels (Case 2). The modelled patch currents when additionally considering membrane damage (Case 3) were less hyperpolarised (the extent of which decreases with an increase of $\tilde{\varepsilon}$ ), producing the same tendencies as the experimental observations. Note that the simulated dynamics are approximately an order of magnitude faster compared to rat F11 cells; this is expected as rat F11 cells are known to have a slow deactivation dynamics [29], not necessarily captured adequately by the Hodgkin-Huxley model, originally calibrated on the squid giant axon [34]. Qualitatively, however, the model is able to capture the general trend of membrane stretch induced signal alteration, with a best fit for $\tilde{\varepsilon} \simeq 0.2$. Additionally, the sole consideration of cell geometrical deformation and ion channel densities are not sufficient, and other mechanical-electrophysiological phenomena such as the membrane damage model used here [19] must be considered. 


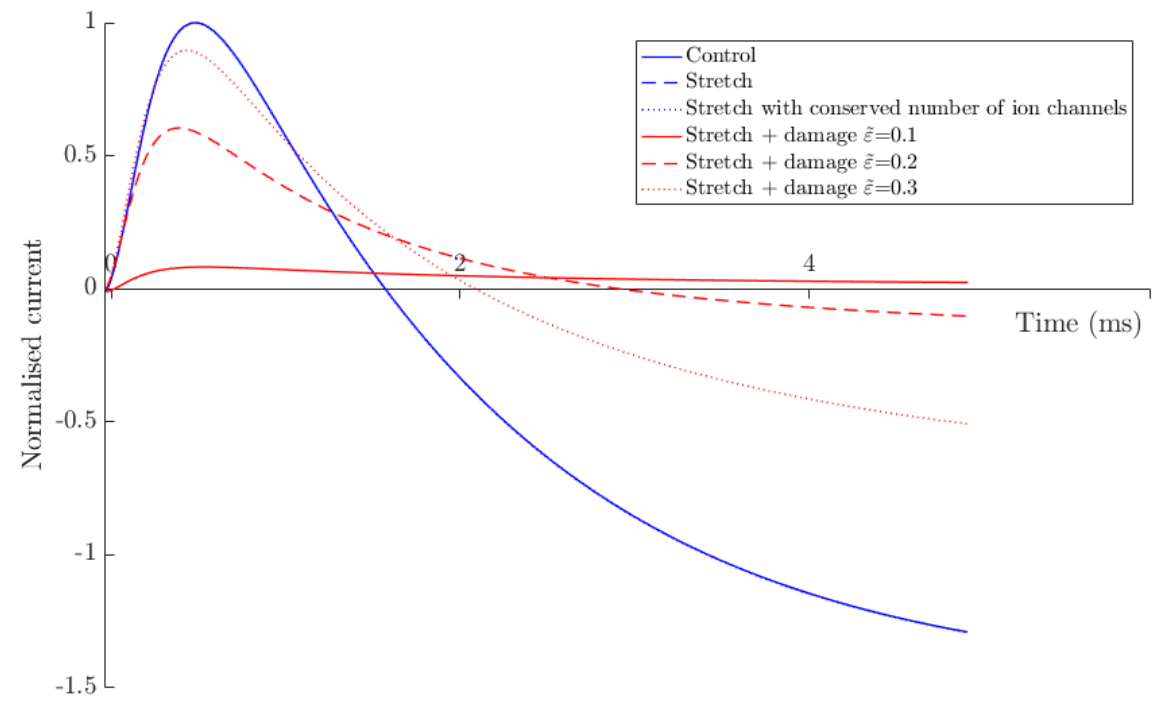

Figure 4: Current from whole cell voltage clamp of $0 \mathrm{mV}$ of control cell without stretch, stretch only (Case 1), stretch with conserved number of channels (Case 2) and stretch with damage for $\tilde{\varepsilon} \in\{0.1,0.2,0.3\}$ (Case 3 ). Note that all blue curves overlap.

\section{Axonal indentation}

The main purpose of this section is to study the AP propagation alteration in an idealised case of spinal dislocation. In such case, the axons can be indented or laterally displaced, e.g., by the shearing movement of one vertebrae, or fragment of bones. Such dislocation leads to a state of loading mixing simultaneously tension and compression. Note also that several cell and tissue scale experiments have used indentation-like setups to approximate axon stretch and mild TBI $[7,5,35]$. The three different mechanicalelectrophysiological alteration mechanisms detailed in Section 3.2.3 were also considered for this study.

\subsection{Simulation setup}

An idealised myelinated axon with two nodes and internodes is considered here. The first node is $2 \mu \mathrm{m}$ long, the second node is $10 \mu \mathrm{m}$ long, and both internodes are $600 \mu \mathrm{m}$ long. The resulting geometry was discretised with 16,638 quadratic tetrahedral elements. Two simulations were considered where a downward pressure of $500 \mathrm{~Pa}$ was applied for 0.05 us on the upper 
parts of the second node and neighbouring internodal region, respectively, see Figure 5. The ends of the axons were mechanically fixed in the horizontal direction, and the longitudinal lines running at the base of the axons were fixed vertically except for the segment being indented. In terms of electrical boundary conditions, the right face was clamped at $-65 \mathrm{mV}$ while a ramp from $-65 \mathrm{mV}$ to $0 \mathrm{mV}$ was applied to the left face over $0.1 \mathrm{~ms}$ after the mechanical loading step (from 0.05 to $0.15 \mathrm{~ms}$ ) and sustained for an additional $9.85 \mathrm{~ms}$. The total simulation time is $10 \mathrm{~ms}$. The measure point for AP propagation in both cases was at $725 \mu \mathrm{m}$. The mechanical and electrophysiological properties considered here are the same as the ones reported in Section 3.2. The membrane strain threshold $\tilde{\varepsilon}$ was chosen to be 0.1 as in Ref. [19] to ease the comparison with the Neurite simulations.

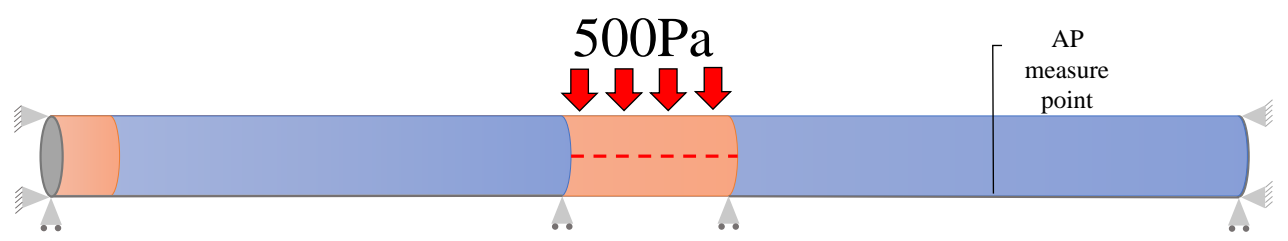

(a)

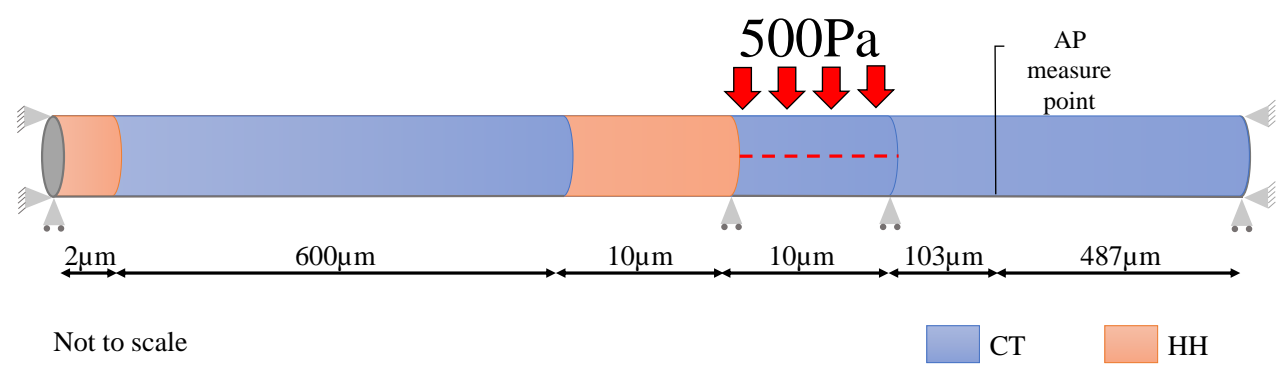

(b)

Figure 5: Axon geometry and boundary condition schematic with mechanical loading at a) the node, b) the internode.

\subsection{Results and discussion}

Figure 6 shows the indentation of the nodal region with a close-up of the site of indentation. Both nodal and internodal indentations result in a 
maximal displacement of $2.84 \mu \mathrm{m}$ at the centre of the segment as the same mechanical properties were chosen for both regions. The post-deformation electrical potential vs. time profiles of the centre-line node of the 3D geometry at the measure point are shown in Figure 7: the AP propagation of an axon without mechanical deformation (green line), with internodal indentation (blue dashed line), with nodal indentation (red dashed line) with nodal indentation and conserved number of ion channels (red dotted line), with nodal indentation and conserved number of damageable ion channels (red full line).

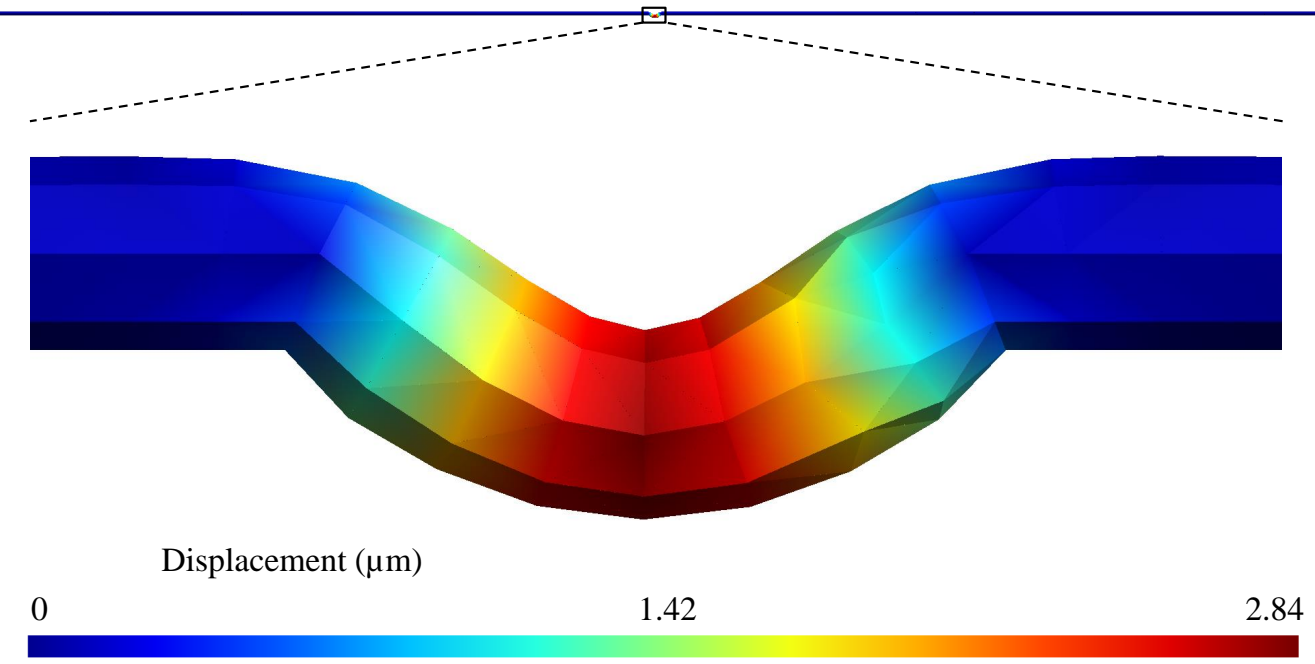

Figure 6: Resultant deformation of $500 \mathrm{~Pa}$ applied on $10 \mu \mathrm{m}$ of axon.

While the indentations applied in this study may not be experimentally realistic, this set of simulations however is useful as an idealised study. Note that the initial rise in potential (up to $-40 \mathrm{mV}$ ) seen in all cases is an artefact of the imposed constant voltage loading used to trigger the AP. The resting potentials of the cases without the damage model are also not fully recovered for the same reason. For the case where the channels' damage model is implemented, the resting potential is affected by the modification of the reversal potentials through the membrane surface strain, see Equation (31).

While the internodal indentation case and the nodal indentation case with conserved number of ion channels (proportional to the original area) 


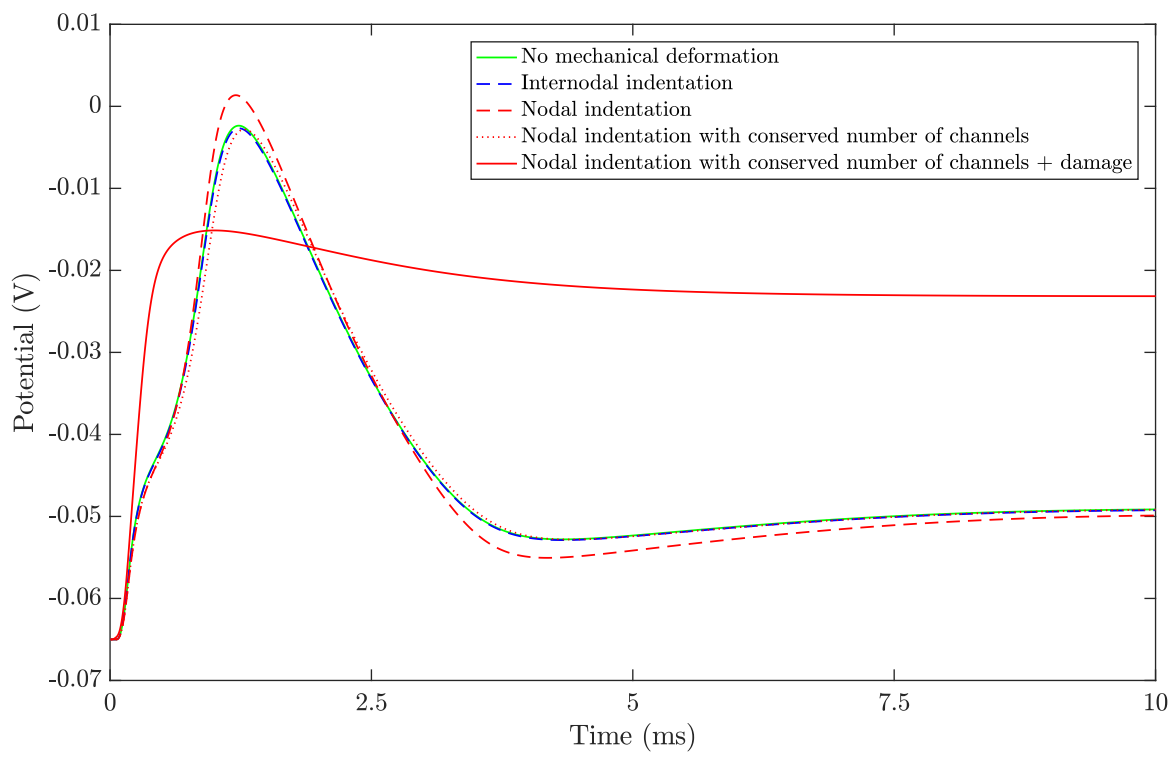

Figure 7: AP propagation comparison between axon without indentation, indentation of internodal segment and nodal segment are presented as a green blue and lines, respectively, with the three model modifications for the nodal indentation.

are very similar to the reference case (no deformation), the default case of nodal indentation (the ion channel number is proportional to the deformed area) sees a noticeable increase in peak amplitude. This is due to an increase in active surface area of the deformed node where HH boundary conditions are applied. For the case of internodal indentation, since the enlarged region is a passive membrane, no remarkable activity change was expected.

In the experimental work of Greer et al. [8], an increase of AP amplitude and a shorter after-hyperpolarisation duration was reported in intact and axotomised (characterised by axonal swelling) pyramidal axons, one and two days after mild TBI induced by a brief fluid pressure pulse. The authors suggested that these observations may be the result of an increase in the number of $\mathrm{Na}^{+}$channels combined with several different underlying $\mathrm{K}^{+}$ channels changes, deeming damaged axons more excitable. These results are in agreement with our model predictions, where long term membrane re-organisation could ensure a recovery of the density of ion channels, and thus increase the membrane activity accordingly to its increase of surface area. Unlike previous efforts $[16,19,22]$, no damage consideration is nee- 
ded to alter the electrical signal. These simulations compare more similarly to the finite difference model of Tekieh et al. [21], where the altered electrophysiology is a direct result of the deformation indentation. However, in their study, a decreased AP amplitude was reported as the nodal geometry becomes depressed. This differs to the results presented here because in an axisymmetric finite difference model, where a depressed nodal region corresponds to a smaller circumferential membrane area, therefore resulting in a lower conductance and, in turn, a decreased AP amplitude. Such discrepancy highlights the need for 3D models when modelling axonal damage.

Based on the theory of coupled left shift in $N a_{V}$ channels (channel type 1.6) reported by Wang et al. [10], Jérusalem et al. [19] proposed a damage model for $N a_{V}$ and $K_{V}$ channels. This model was implemented and presented as the red solid line in Figure 7 . The AP amplitude is significantly decreased by the damage model compared to the other cases. Note that the critical strain for the damage model used here is 0.1 , which is the same as the value reported by Jérusalem et al. [19]. This value was calibrated with tissue scale data, and hence may not be applicable for a model on cellular scale. The aim of this particular result however is to demonstrate the flexibility of this framework to explore different damage mechanisms.

\subsection{Comparison of coupled left shift theory between $1 D$ and 3D models}

In order to further evaluate the difference between 1D and 3D models, the case of axon indentation was modelled in 1D with Neurite and is presented in Figure 8 as a blue dashed line (solid line is without deformation). The axon indentation in 1D was modelled by applying an axial strain of 0.179 (approximated from the centre line of $3 \mathrm{D}$ deformation from the 3D FE simulations shown in Figure 6) to the corresponding node of a Neurite axon simulation. The APs for the 3D FE cases with no mechanical deformation and nodal indentation with conserved damageable ion channel number (i.e., the same underlying model as in Neurite) are shown as red lines in Figure 8.

There is an insignificant difference between the non-indented and the indented axon modelled in $1 \mathrm{D}$, as the indentation equivalent axial stretch is insufficient to cause any alteration in electrophysiology, while the amount of damage present in the 3D formulation is more significant than in the 1D case. This is due to the ability of the 3D formulation to capture local gradient of deformation in a small region, enough to affect the overall electrophysiology. Indeed, while a mean increase in length is equally observed, the 3D model is additionally able to capture non-axisymmetric large tensile strain in the 


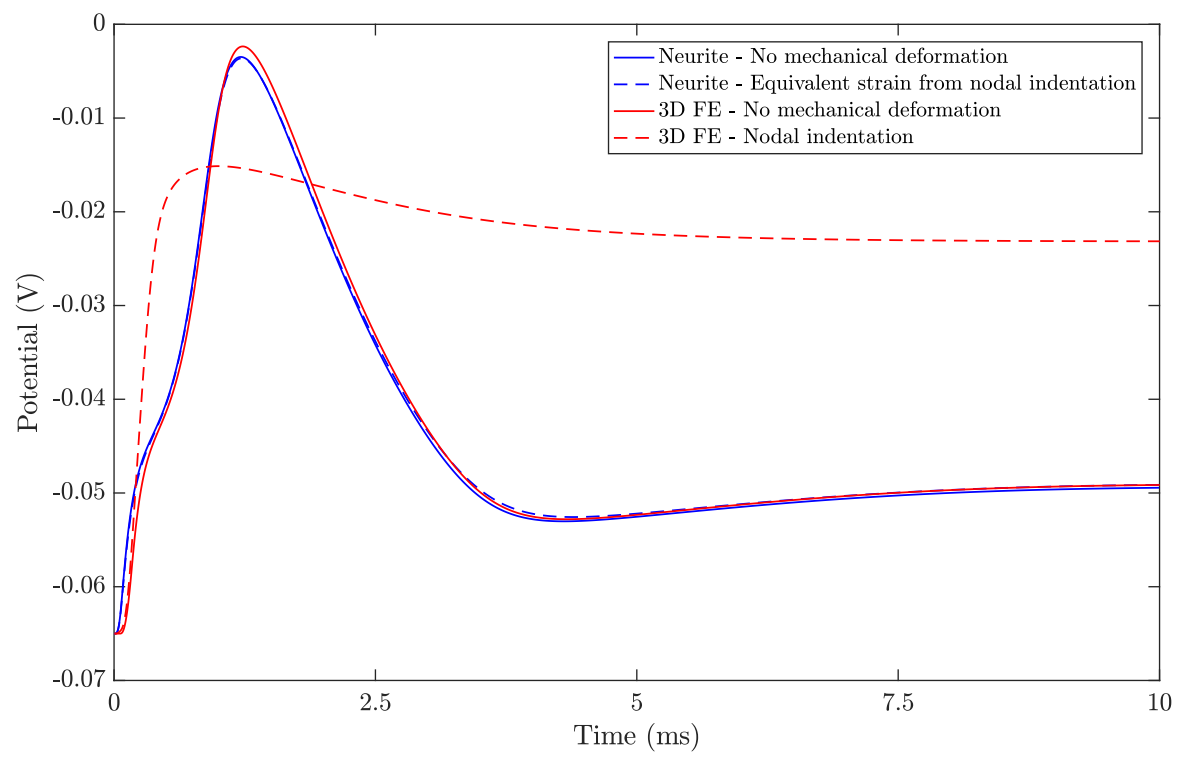

Figure 8: AP propagation comparison between 3D FE and Neurite simulations.

membrane. Overall this idealised axonal dislocation case demonstrates the ability of the proposed framework to capture electrophysiological alterations associated to localised damage.

\section{Conclusion}

In this study a 3D finite element framework for the description of the mechanics and electrophysiology of axons was proposed. In this model, the electrophysiological HH and CT models, respectively, for the nodes of Ranvier and internodes, were introduced as surface boundary conditions directly in the weak form. The model was validated against numerical and experimental results. Its application in an idealised case of axonal dislocation shows that, for a mild indentation, the sole consideration of induced longitudinal stretch following transverse loading of a node of Ranvier is not enough to capture the extent of axonal electrophysiological deficit. In the internodal region such load was not shown to significantly worsen the electrophysiological behaviour when additionally considering the non-axisymmetric components of the loading. 
The ability of this framework to capture electrophysiological changes associated with 3D deformation is especially important as advance mechanicalelectrophysiological experimental work involving 3D spatio-temporal strain field of neurons become widely available [2]. Other future applications of the model include the study of membrane related injury mechanisms such as mechanoporation $[7,11,9,6]$, coupled left shift of voltage gated ion channels [10] and re-organisation of paranodal junctions [17]. Further development of this framework will also allow for the modelling of electrophysiological driven membrane morphology change observed during patch clamp studies [18]. It is finally worth noting that an extension of the model to cellular network would require the implementation of cellular synapses both mechanically and electrophysiologically; additional coupling between cells and substrate or extracellular matrix might also be required.

\section{Acknowledgements}

M.T.K., M.B. and A.J. acknowledge funding from the European Union's Seventh Framework Programme (FP7 20072013) ERC Grant Agreement No.306587. H.Y. would like to acknowledge China Regenerative Medicine Limited (CRMI) for funding and the EPSRC DTP (Award no. 1514540) for F.B.'s studentship.

[1] A. Goriely, M. G. D. Geers, G. A. Holzapfel, J. Jayamohan, A. Jérusalem, S. Sivaloganathan, W. Squier, J. A. W. van Dommelen, S. Waters, E. Kuhl, Mechanics of the brain: perspectives, challenges, and opportunities, Biomech Model Mechanobiol 14 (5) (2015) 931-965.

[2] E. Bar-Kochba, M. T. Scimone, J. B. Estrada, C. Franck, Strain and rate-dependent neuronal injury in a $3 \mathrm{D}$ in vitro compression model of traumatic brain injury, Sci Rep 6 (2016) 30550.

[3] Y. C. Chen, D. H. Smith, D. F. Meaney, In-vitro approaches for studying blast-induced traumatic brain injury, J Neurotrauma 26 (6) (2009) 86176 .

[4] G. C. Magou, B. J. Pfister, J. R. Berlin, Effect of acute stretch injury on action potential and network activity of rat neocortical neurons in culture, Brain Res 1624 (2015) 525-535. 
[5] G. C. Magou, Y. Guo, M. Choudhury, L. Chen, N. Hususan, S. Masotti, B. J. Pfister, Engineering a high throughput axon injury system, J Neurotrauma 28 (11) (2011) 2203-2218.

[6] W. Sun, Y. Fu, Y. Shi, J.-X. Cheng, P. Cao, R. Shi, Paranodal myelin damage after acute stretch in guinea pig spinal cord, J Neurotrauma 29 (3) (2012) 611-619.

[7] R. Shi, J. Whitebone, Conduction deficits and membrane disruption of spinal cord axons as a function of magnitude and rate of strain, J Neurophysiol 95 (2006) 3384-3390.

[8] J. E. Greer, J. T. Povlishock, K. M. Jacobs, Electrophysiological abnormalities in both axotomized and nonaxotomized pyramidal neurons following mild traumatic brain injury, J Neurosci 32 (19) (2012) 66826687.

[9] H. Ouyang, W. Sun, Y. Fu, J. Li, J.-X. Cheng, E. Nauman, R. Shi, Compression induces acute demyelination and potassium channel exposure in spinal cord, J Neurotrauma 27 (6) (2010) 1109-1120.

[10] J. A. Wang, W. Lin, T. Morris, U. Banderali, P. F. Juranka, C. E. Morris, Membrane trauma and $\mathrm{Na}^{+}$leak from Nav1.6 channels, Am J Physiol Cell Physiol 297 (4) (2009) C823-C834.

[11] H. Ouyang, B. Galle, J. Li, E. Nauman, R. Shi, Biomechanics of spinal cord injury: a multimodal investigation using ex vivo guinea pig spinal cord white matter, J Neurotrauma 25 (1) (2008) 19-29.

[12] B. J. Pfister, D. P. Bonislawski, D. H. Smith, A. S. Cohen, Stretch-grown axons retain the ability to transmit active electrical signals, FEBS Lett 580 (14) (2006) 3525-3531.

[13] W. H. Kang, W. Cao, O. Graudejus, T. P. Patel, S. Wagner, D. F. Meaney, B. r. Morrison, Alterations in hippocampal network activity after in vitro traumatic brain injury, J Neurotrauma 32 (13) (2015) 1011-1019.

[14] W. H. Kang, B. Morrison, Predicting changes in cortical electrophysiological function after in vitro traumatic brain injury, Biomech Model Mechanobiol 14 (5) (2015) 1033-1044. 
[15] C. F. Babbs, R. Shi, Subtle paranodal injury slows impulse conduction in mathematical model of myelinated axons, PLoS One 8 (7) (2013) $1-11$.

[16] P. A. Boucher, B. Joós, C. E. Morris, Coupled left-shift of Nav channels: Modeling the $\mathrm{Na}^{+}$-loading and dysfunctional excitability of damaged axons, J Comput Neurosci 33 (2) (2012) 301-319.

[17] V. Volman, L. J. Ng, Primary paranode demyelination modulates slowly developing axonal depolarization in a model of axonal injury, J Comput Neurosci 37 (3) (2014) 439-457.

[18] T. M. Suchyna, V. S. Markin, F. Sachs, Biophysics and structure of the patch and the gigaseal, Biophys J 97 (3) (2009) 738-747.

[19] A. Jérusalem, J. A. García-Grajales, A. Merchán-Pérez, J. M. Peña, A computational model coupling mechanics and electrophysiology in spinal cord injury, Biomech Model Mechanobiol 13 (4) (2014) 883-896.

[20] C. S. Drapaca, An electromechanical model of neuronal dynamics using Hamilton's principle, Front Cell Neurosci 9 (2015) 271.

[21] T. Tekieh, S. Shahzadi, H. Rafii-Tabar, P. Sasanpour, Are deformed neurons electrophysiologically altered? A simulation study, Curr Appl Phys 16 (2016) 1413-1417.

[22] I. Cinelli and M. Destrade and M. Duffy and P. McHugh, Electromechanical response of a 3D nerve bundle model to mechanical loads leading to ixonal injury, Int J Numer Method Biomed Eng (2017) In press.

[23] Simulia, Abaqus 6.10 (2010).

[24] C. Geuzaine, J. F. Remacle, Gmsh: A 3D finite element mesh generator with built-in pre- and post-processing facilities, Int J Numer Meth Eng 79 (11) (2009) 1309-1331.

[25] L. Wu, D. Tjahjanto, G. Becker, A. Makradi, A. Jérusalem, L. Noels, A micro-meso-model of intra-laminar fracture in fiber-reinforced composites based on a discontinuous Galerkin/cohesive zone method, Eng Fract Mech 104 (2013) 162-183. 
[26] G. Cheng, R.-h. Kong, L. M. Zhang, J. N. Zhang, Mitochondria in traumatic brain injury and mitochondrial-targeted multipotential therapeutic strategies, Br J Pharmacol 167 (2012) 699-719.

[27] M. A. Avery, T. M. Rooney, J. D. Pandya, T. M. Wishart, T. H. Gillingwater, J. W. Geddes, P. Sullivan, M. R. Freeman, $W l d^{S}$ prevents axon degeneration through increased mitochondrial flux and enhanced mitochondrial $\mathrm{Ca}^{2+}$ buffering, Curr Biol 22 (2012) 596-600.

[28] J. A. García-Grajales, G. Rucabado, A. García-Dopico, J. Peña, A. Jérusalem, Neurite, a finite difference large scale parallel program for the simulation of electrical signal propagation in neurites under mechanical loading, PLoS One 10 (2) (2015) 1-22.

[29] F. Jow, L. He, A. Kramer, J. Hinson, M. R. Bowlby, J. Dunlop, K. W. Wang, Validation of DRG-like F11 cells for evaluation of KCNQ/Mchannel modulators, Assay Drug Dev Technol 4 (1) (2006) 49-56.

[30] S. F. Fan, K. F. Shen, M. A. Scheideler, S. M. Crain, F11 neuroblastoma $\mathrm{x}$ DRG neuron hybrid cells express inhibitory/.1- and 6-opioid receptors which increase voltage-dependent $\mathrm{K}+$ currents upon activation, Brain Res 590 (1992) 329-222.

[31] F. Bianchi, J. H. George, M. Malboubi, A. Jerusalem, M. S. Thompson, H. Ye, Engineering a uniaxial substrate-stretching device for simultaneous electrophysiology and imaging of strained peripheral neurons, In Preparation.

[32] M. Malboubi, Y. Gu, K. Jiang, Characterization of surface properties of glass micropipettes using SEM stereoscopic technique, Microelectron Eng 88 (8) (2011) 2666-2670.

[33] J. Loverde, B. Pfister, Developmental axon stretch stimulates neuron growth while maintaining normal electrical activity, intracellular calcium flux, and somatic morphology, Front Cell Neurosci 9 (2015) 308.

[34] A. Hodgkin, A. Huxley, A quantitative description of membrane current and its application to conduction and excitation in nerve, J Physiol 117 (1952) 500-544. 
[35] D. H. Smith, J. A. Wolf, T. A. Lusardi, V. M. Lee, D. F. Meaney, High tolerance and delayed elastic response of cultured axons to dynamic stretch injury, J Neurosci 19 (11) (1999) 4263-4269.

[36] C. Koch, Biophysics of computation:, Oxford University Press, 1999.

\section{Appendix A. Hodgkin-Huxley ion channel activity}

The dynamic conductances $G_{N a}$ and $G_{K}$ are functions of the time-dependent membrane potential $V$, and two reference values $\bar{G}_{N a}$ and $\bar{G}_{K}[34,36]$, see Table A.3. In this table, the dimensionless activation $(m$ and $n)$ and inactivation $(h)$ states describe the evolution of the corresponding conductances as a function of the rate constants $\alpha_{k}$ and $\beta_{k}$ for $k \in\{m, h, n\}$. The states need to be simultaneously open in a given configuration $(3 \mathrm{~m}$ 's and $1 h$ for $N a_{v} ; 4 n$ 's for $K_{v}$ ) to allow for the full opening of the gate, see Refs. [34] and [36] for further information. 


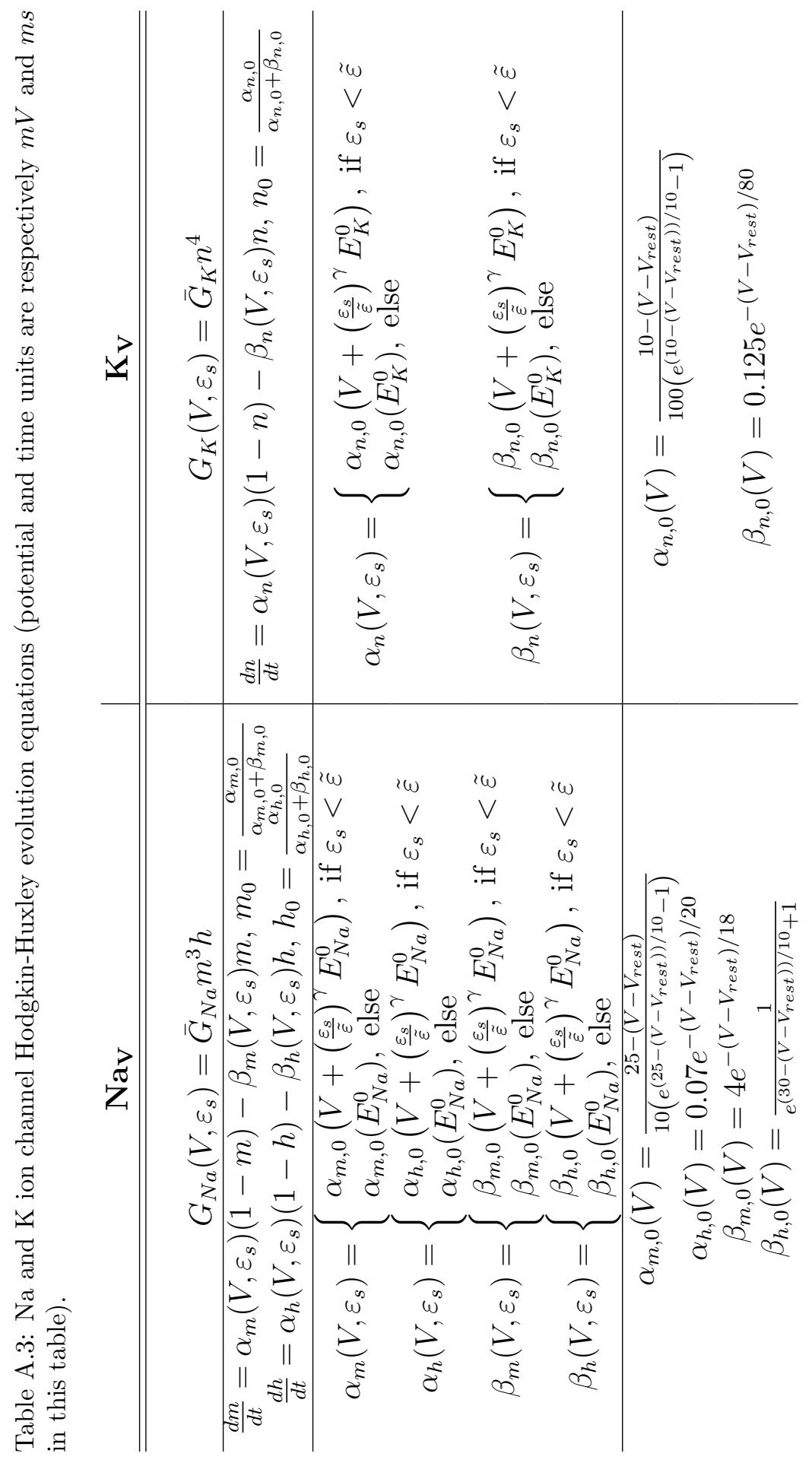


Appendix A.1. $\frac{\partial f_{C T}}{\partial V}$

Recalling Equations (13) and (14):

$$
\begin{aligned}
f_{C T}\left(V, \frac{\partial V}{\partial t}, \varepsilon_{s}\right)=-\rho_{c} & \left(\frac{C_{m} C_{m y}}{h_{m} C_{m y}+n_{m y} h_{m y} C_{m}} \frac{\partial V}{\partial t}\right. \\
& \left.+\frac{1}{h_{m} \rho_{m}+n_{m y} h_{m y} \rho_{m y}}\left(V-V_{\text {rest }}\right)\right)
\end{aligned}
$$

In incremental form with respect to $V$ at time $t^{i+1}$, this equation reads:

$$
\begin{aligned}
f_{C T}\left(V^{\alpha}, \frac{V^{i+1}-V^{i}}{\Delta t}, \varepsilon_{s}\right)=-\rho_{c} & \left(\frac{C_{m} C_{m y}}{h_{m} C_{m y}+n_{m y} h_{m y} C_{m}} \frac{V^{i+1}-V^{i}}{\Delta t}\right. \\
& \left.+\frac{1}{h_{m} \rho_{m}+n_{m y} h_{m y} \rho_{m y}}\left(V^{\alpha}-V_{\text {rest }}\right)\right)
\end{aligned}
$$

where $V^{i+1}, V^{i}$ are, respectively, the values of $V$ at $t^{i+1}$ and $t^{i}$, where $\Delta t=$ $t^{i+1}-t^{i}$ is the time step, $\alpha$ is a parameter specifying the intermediary time between $t^{i+1}$ and $t^{i}$, and where:

$$
V^{\alpha}=(1-\alpha) V^{i}+\alpha V^{i+1}
$$

Ultimately, the derivation of Equation (A.2) with respect to $V^{i+1}$ yields:

$$
\left.\frac{\partial f_{C T}}{\partial V}\right|_{V=V^{i+1}}=-\frac{\rho_{c}}{\Delta t}\left(\frac{C_{m} C_{m y}}{h_{m} C_{m y}+n_{m y} h_{m y} C_{m}}+\frac{\alpha \Delta t}{h_{m} \rho_{m}+n_{m y} h_{m y} \rho_{m y}}\right)
$$

\section{Appendix A.2. $\frac{\partial \boldsymbol{f}_{H H}}{\partial \boldsymbol{V}}$}

Recalling Equations (13) and (14):

$$
\begin{aligned}
f_{H H}\left(V, \frac{\partial V}{\partial t}, \varepsilon_{s}\right)=\frac{-\rho_{c}}{h_{m}}\left(C_{m} \frac{\partial V}{\partial t}\right. & +G_{N a}\left(V-E_{N a}\left(\varepsilon_{s}\right)\right) \\
& \left.+G_{K}\left(V-E_{K}\left(\varepsilon_{s}\right)\right)+G_{L}\left(V-E_{L}\right)\right)
\end{aligned}
$$


In incremental form with respect to $V$ at time step $t^{i+1}$, this equation reads:

$$
\begin{aligned}
f_{H H}\left(V^{\alpha}, \frac{V^{i+1}-V^{i}}{\Delta t}, \varepsilon_{s}\right)=\frac{-\rho_{c}}{h_{m}}\left(C_{m} \frac{V^{i+1}-V^{i}}{\Delta t}\right. & +G_{N a}\left(V^{\alpha}, \varepsilon_{s}\right)\left(V^{\alpha}-E_{N a}\left(\varepsilon_{s}\right)\right) \\
& +G_{K}\left(V^{\alpha}, \varepsilon_{s}\right)\left(V^{\alpha}-E_{K}\left(\varepsilon_{s}\right)\right) \\
& \left.+G_{L}\left(V^{\alpha}, \varepsilon_{s}\right)\left(V^{\alpha}-E_{L}\left(V^{\alpha}, \varepsilon_{s}\right)\right)\right)
\end{aligned}
$$

Ultimately, one has

$$
\begin{aligned}
\left.\frac{\partial f_{H H}}{\partial V}\right|_{V=V^{i+1}}=\frac{-\rho_{c}}{h_{m}}\left(\frac{C_{m}}{\Delta t}\right. & +\alpha\left(G_{N a}\left(V^{\alpha}, \varepsilon_{s}\right)+G_{K}\left(V^{\alpha}, \varepsilon_{s}\right)+G_{L}\right) \\
& +\left.\frac{\partial G_{N a}\left(V^{\alpha}, \varepsilon_{s}\right)}{\partial V}\right|_{V=V^{i+1}}\left(V^{\alpha}-E_{N a}\left(\varepsilon_{s}\right)\right) \\
& +\left.\frac{\partial G_{K}\left(V^{\alpha}, \varepsilon_{s}\right)}{\partial V}\right|_{V=V^{i+1}}\left(V^{\alpha}-E_{K}\left(\varepsilon_{s}\right)\right) \\
& \left.-\left.G_{L} \frac{\partial E_{L}\left(V^{\alpha}, \varepsilon_{s}\right)}{\partial V}\right|_{V=V^{i+1}}\right)
\end{aligned}
$$

where, using Table A.3,

$$
\left\{\begin{array}{l}
\left.\frac{\partial G_{N a}\left(V^{\alpha}, \varepsilon_{s}\right)}{\partial V}\right|_{V=V^{i+1}}=\alpha \bar{G}_{N a}\left(\left.3 m_{\alpha}^{2} h_{\alpha} \frac{\partial m}{\partial V}\right|_{V=V^{\alpha}}+\left.m_{\alpha}^{3} \frac{\partial h}{\partial V}\right|_{V=V^{\alpha}}\right) \\
\left.\frac{\partial G_{K}\left(V^{\alpha}, \varepsilon_{s}\right)}{\partial V}\right|_{V=V^{i+1}}=\left.4 \alpha n_{\alpha}^{3} \bar{G}_{K} \frac{\partial n}{\partial V}\right|_{V=V^{\alpha}}
\end{array}\right.
$$

where forward Euler approximation is used as follows:

$$
\left\{\begin{array}{l}
m_{\alpha} \approx m_{i}+\left.\alpha \Delta t \frac{d m}{d t}\right|_{V=V^{i}} \\
h_{\alpha} \approx h_{i}+\left.\alpha \Delta t \frac{d h}{d t}\right|_{V=V^{i}} \\
n_{\alpha} \approx n_{i}+\left.\alpha \Delta t \frac{d n}{d t}\right|_{V=V^{i}}
\end{array}\right.
$$

where the temporal derivatives of $m, h$ and $n$ at time step $t_{i}$ are directly obtained from Table A.3. The remaining terms of Equation (A.8), i.e., $\left.\frac{\partial m}{\partial V}\right|_{V=V^{\alpha}}$, $\left.\frac{\partial h}{\partial V}\right|_{V=V^{\alpha}}$ and $\left.\frac{\partial n}{\partial V}\right|_{V=V^{\alpha}}$, can be evaluated numerically. Alternatively, under loading slow enough so that the variation of $m, n$ and $h$ are mainly driven 
by variation in $V$ and not in $\varepsilon_{s}$, the following approximation can be made:

$$
\left\{\begin{array}{l}
\left.\left.\frac{\partial m}{\partial V}\right|_{V=V^{\alpha}} \approx \frac{\Delta t}{V^{i+1}-V^{i}} \frac{d m}{d t}\right|_{V=V^{i}} \\
\left.\left.\frac{\partial h}{\partial V}\right|_{V=V^{\alpha}} \approx \frac{\Delta t}{V^{i+1}-V^{i}} \frac{d h}{d t}\right|_{V=V^{i}} \\
\left.\left.\frac{\partial n}{\partial V}\right|_{V=V^{\alpha}} \approx \frac{\Delta t}{V^{i+1}-V^{i}} \frac{d n}{d t}\right|_{V=V^{i}}
\end{array}\right.
$$

This last assumption is naturally a priori violated under very high strain rate loading cases such as blast loadings.

\section{Appendix A.3. $\frac{\partial f_{C T}}{\partial u_{k b}}$}

Under the assumption that the CT permittivity, resistivities and membrane/myelin layer thicknesses are independent of the deformation because of the geometrical dependency, the derivation of Equation (A.1) yields:

$$
\frac{\partial f_{C T}}{\partial u_{k b}}=0
$$

Note that this would not be the case if involving damage-driven capacity and/or resistivity alteration.

\section{Appendix A.4. $\frac{\partial \boldsymbol{f}_{\boldsymbol{H}}}{\partial \boldsymbol{u}_{\boldsymbol{H}}}$}

While $\mathrm{HH}$ membrane and leak permittivity, resistivities and conductivities are independent of the deformation, the ion channels conductivities and potentials are deformation dependent. The derivation of $f_{H H}$ with respect to $u_{k b}$ thus yields:

$$
\begin{aligned}
\frac{\partial f_{H H}}{\partial u_{k b}}=\frac{-\rho_{c}}{h_{m}} \frac{\partial \varepsilon_{s}}{\partial u_{k b}}( & \frac{\partial G_{N a}\left(V, \varepsilon_{s}\right)}{\partial \varepsilon_{s}}\left(V-E_{N a}\left(\varepsilon_{s}\right)\right) \\
& +\frac{\partial G_{K}\left(V, \varepsilon_{s}\right)}{\partial \varepsilon_{s}}\left(V-E_{K}\left(\varepsilon_{s}\right)\right) \\
& -G_{N a}\left(V, \varepsilon_{s}\right) \frac{\partial E_{N a}\left(\varepsilon_{s}\right)}{\partial \varepsilon_{s}} \\
& \left.-G_{K}\left(V, \varepsilon_{s}\right) \frac{\partial E_{K}\left(\varepsilon_{s}\right)}{\partial \varepsilon_{s}}\right)
\end{aligned}
$$


where, using Table A.3, ${ }^{1}$

$$
\left\{\begin{array}{l}
\frac{\partial G_{N a}\left(V, \varepsilon_{s}\right)}{\partial \varepsilon_{s}}=\bar{G}_{N a}\left(3 m^{2} h \frac{\partial m}{\partial \varepsilon_{s}}+m^{3} \frac{\partial h}{\partial \varepsilon_{s}}\right) \\
\frac{\partial G_{K}\left(V, \varepsilon_{s}\right)}{\partial \varepsilon_{s}}=4 n^{3} \bar{G}_{K} \frac{\partial n}{\partial \varepsilon_{s}}
\end{array}\right.
$$

and

$$
\left\{\begin{array}{l}
\frac{\partial E_{N a}\left(\varepsilon_{s}\right)}{\partial \varepsilon_{s}}= \begin{cases}-\frac{\gamma}{\tilde{\varepsilon}}\left(\frac{\varepsilon_{s}}{\tilde{\varepsilon}}\right)^{\gamma-1} E_{N a}^{0}, & \text { if } \varepsilon_{s}<\tilde{\varepsilon} \\
0, & \text { else. }\end{cases} \\
\frac{\partial E_{K}\left(\varepsilon_{s}\right)}{\partial \varepsilon_{s}}= \begin{cases}-\frac{\gamma}{\tilde{\varepsilon}}\left(\frac{\varepsilon_{s}}{\tilde{\varepsilon}}\right)^{\gamma-1} E_{K}^{0}, & \text { if } \varepsilon_{s}<\tilde{\varepsilon} \\
0, & \text { else. }\end{cases}
\end{array}\right.
$$

In Equation (A.13), $\frac{\partial m}{\partial \varepsilon_{s}}, \frac{\partial h}{\partial \varepsilon_{s}}$ and $\frac{\partial n}{\partial \varepsilon_{s}}$ can be evaluated numerically. $\varepsilon_{s}$ is defined in Appendix A.6

\section{Appendix A.5. $\frac{\partial \boldsymbol{n}_{\boldsymbol{i}}}{\partial \boldsymbol{u}_{\boldsymbol{k}}}$}

For any element face belonging to the domain boundary $\partial \Omega_{0}$, such as the grey area in Figure A.9, the tangent basis vectors $\mathbf{a}_{\mathbf{1}}$ and $\mathbf{a}_{\mathbf{2}}$ are defined by:

$$
\mathbf{a}_{\alpha}(\boldsymbol{\xi})=\sum_{a=1}^{n_{s}} \mathbf{x}_{a}^{s} \mathcal{N}_{a, \alpha}^{s}(\boldsymbol{\xi}), \forall \alpha \in\{1,2\}
$$

where $\mathcal{N}_{a, \alpha}^{s}(\boldsymbol{\xi})$ are the corresponding shape functions derivatives with respect to the natural direction $\alpha, \boldsymbol{\xi}$ being the coordinate vector in the surface element isoparametric reference frame. The normalised normal vector is then given by:

$$
\mathbf{n}=\frac{\mathbf{a}_{1} \times \mathbf{a}_{2}}{\left\|\mathbf{a}_{1} \times \mathbf{a}_{2}\right\|}
$$

As a consequence, one has:

$$
\frac{\partial n_{i}}{\partial u_{k b}}=\frac{\partial n_{i}}{\partial x_{k b}}=\underbrace{\frac{1}{\left\|\mathbf{a}_{1} \times \mathbf{a}_{2}\right\|} \frac{\partial\left[\left(\mathbf{a}_{1} \times \mathbf{a}_{2}\right) \cdot \mathbf{e}_{i}\right]}{\partial x_{k b}}}_{A}+\underbrace{\left[\left(\mathbf{a}_{1} \times \mathbf{a}_{2}\right) \cdot \mathbf{e}_{i}\right] \frac{\partial}{\partial x_{k b}}\left(\frac{1}{\left\|\mathbf{a}_{1} \times \mathbf{a}_{2}\right\|}\right)}_{B}
$$

\footnotetext{
${ }^{1}$ Note that under the same assumption as in Equation (A.10), both following terms can be considered equal to 0 .
} 


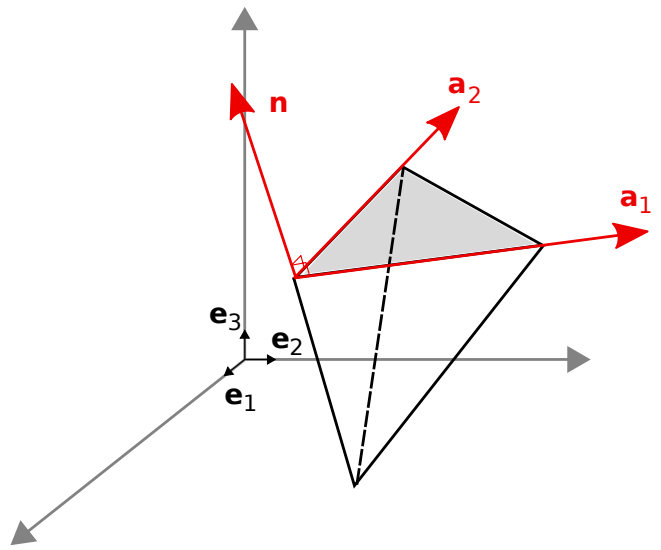

Figure A.9: Surface element: tangent basis vector $\mathbf{a}_{1}$ and $\mathbf{a}_{2}$, and normal vector $\mathbf{n}$ in the global reference frame defined by the basis vectors $\left(\mathbf{e}_{1}, \mathbf{e}_{2}, \mathbf{e}_{3}\right)$.

- Using the Levi-Vicita-or permutation-symbol, $\epsilon_{i j k}$, one obtains:

$$
A=\frac{1}{\left\|\mathbf{a}_{1} \times \mathbf{a}_{2}\right\|} \frac{\partial\left(\epsilon_{i j l} a_{1 j} a_{2 l}\right)}{\partial x_{k b}}=\frac{\epsilon_{i j l}}{\left\|\mathbf{a}_{1} \times \mathbf{a}_{2}\right\|}\left(a_{1 j} \frac{\partial a_{2 l}}{\partial x_{k b}}+a_{2 l} \frac{\partial a_{1 j}}{\partial x_{k b}}\right)
$$

Based on Equation (A.15),

$$
\left\{\begin{array}{l}
\frac{\partial a_{1 j}}{\partial x_{k b}}=\delta_{j k} \mathcal{N}_{b, 1}^{s} \\
\frac{\partial a_{2 l}}{\partial x_{k b}}=\delta_{l k} \mathcal{N}_{b, 2}^{s}
\end{array}\right.
$$

Noting that $\epsilon_{i j l}=-\epsilon_{j i l}$, Equation (A.18) can thus be rewritten as:

$$
A=\frac{\epsilon_{i k l}}{\left\|\mathbf{a}_{1} \times \mathbf{a}_{2}\right\|}\left(a_{2 l} \mathcal{N}_{b, 1}^{s}-a_{1 l} \mathcal{N}_{b, 2}^{s}\right)
$$

which can also be rewritten as

$$
A=\frac{\epsilon_{k l q}}{\left\|\mathbf{a}_{1} \times \mathbf{a}_{2}\right\|}\left(a_{2 l} \mathcal{N}_{b, 1}^{s}-a_{1 l} \mathcal{N}_{b, 2}^{s}\right) \delta_{i q}
$$

- One has:

$$
B=-\left[\left(\mathbf{a}_{1} \times \mathbf{a}_{2}\right) \cdot \mathbf{e}_{i}\right] \frac{\partial\left\|\mathbf{a}_{1} \times \mathbf{a}_{2}\right\|}{\partial x_{k b}} \frac{1}{\left\|\mathbf{a}_{1} \times \mathbf{a}_{2}\right\|^{2}}
$$


and by noting that the derivative of a vector norm can be written as $\|\mathbf{u}\|^{\prime}=\frac{\mathbf{u} \cdot \mathbf{u}^{\prime}}{\|\mathbf{u}\|}, B$ can be rewritten as

$$
B=-\frac{n_{i}}{\left\|\mathbf{a}_{1} \times \mathbf{a}_{2}\right\|} \mathbf{n} \cdot \frac{\partial\left(\mathbf{a}_{\mathbf{1}} \times \mathbf{a}_{\mathbf{2}}\right)}{\partial x_{k b}}=-\frac{n_{i} \epsilon_{k l q}}{\left\|\mathbf{a}_{1} \times \mathbf{a}_{2}\right\|}\left(a_{2 l} \mathcal{N}_{b, 1}^{s}-a_{1 l} \mathcal{N}_{b, 2}^{s}\right) n_{q}
$$

making use of the derivation of $A$.

Finally, gathering Equations (A.21) and (A.23):

$$
\frac{\partial n_{i}}{\partial u_{k b}}=\frac{\epsilon_{k l q}}{\left\|\mathbf{a}_{1} \times \mathbf{a}_{2}\right\|}\left(a_{2 l} \mathcal{N}_{b, 1}^{s}-a_{1 l} \mathcal{N}_{b, 2}^{s}\right)\left(\delta_{i q}-n_{i} n_{q}\right)
$$

Appendix A.6. $\varepsilon_{s}$

The surface strain $\varepsilon_{s}$ can be defined as:

$$
\varepsilon_{s}=\frac{\mathbf{a}_{1} \times \mathbf{a}_{2}}{\left\|\mathbf{A}_{1} \times \mathbf{A}_{2}\right\|}-1
$$

where $\mathbf{A}_{1}$ and $\mathbf{A}_{2}$ are the counterparts of $\mathbf{a}_{1}$ and $\mathbf{a}_{2}$, respectively, in the reference configuration.

\section{Appendix B. Electrophysiological validation}

The CT and HH equations implementations were first verified separately using a cylinder with a diameter of $3 \mu \mathrm{m}$ and lengths of $100 \mu \mathrm{m}$ and $600 \mu \mathrm{m}$, respectively. Dirichlet boundary conditions were applied to both ends of the axon: $0 \mathrm{mV}$ on the left hand side and $-65 \mathrm{mV}$ (the resting potential) on the right hand side. $\mathrm{CT}$ and $\mathrm{HH}$ boundary conditions were applied to the envelope of the cylinder representing the axonal membrane.

The electrophysiological parameters are the same as in Ref. [19, 28], except for the membrane resistivity whose value was arbitrarily reduced thousandfold and the number of myelin layers was set to zero for the CT simulations so as to artificially accentuate the non-linear effects of membrane current leak and better confirm the scheme convergence, see Table 1.

Tables B.4 and B.5 summarise the spatial and time discretisation parameters used in all three programs. Spatial and temporal convergences were verified for all three cases. 


\begin{tabular}{llll}
\hline & Neurite & 1D FE & 3D FE \\
\hline \hline Number of elements & 2000 & 80 & 134 (along x) \\
Element length $(\mathrm{m})$ & $5 \times 10^{-8}$ & $125 \times 10^{-8}$ & $75 \times 10^{-8}$ \\
Time step size $(\mathrm{s})$ & $2.5 \times 10^{-11}$ & $1 \times 10^{-6}$ & $5 \times 10^{-6}$ \\
Relative NR tolerance & $\mathrm{N} / \mathrm{A}$ & $1 \times 10^{-8}$ & $1 \times 10^{-8}$ \\
\hline
\end{tabular}

Table B.4: CT simulation parameters.

\begin{tabular}{llll}
\hline & Neurite & 1D FE & 3D FE \\
\hline \hline Number of elements & 500 & 80 & 800 (along x) \\
Element length $(\mathrm{m})$ & $120 \times 10^{-8}$ & $125 \times 10^{-8}$ & $75 \times 10^{-8}$ \\
Time step size(s) & $1.8 \times 10^{-8}$ & $1 \times 10^{-8}$ & $1 \times 10^{-5}$ \\
Relative NR tolerance & $\mathrm{N} / \mathrm{A}$ & $1 \times 10^{-8}$ & $1 \times 10^{-6}$ \\
\hline
\end{tabular}

Table B.5: HH simulation parameters.

The resulting steady-state voltage field in the absence of mechanical deformation (taken here at $50 \mu \mathrm{s}$ ) as predicted by the 3D FE model with CT boundary conditions is presented in Figure B.10a. A plot showing the voltage profiles along the axon computed by all three codes is presented in Figure B.10b, where the 3D FE profile plot was generated by extracting the values of the centre-line nodes of the 3D cylinder geometry. The effect of leakage in the CT equation was associated with the system's state-space constant: the ratio of transverse to axial resistivities [36].

A longer geometry and simulation time was necessary for $\mathrm{HH}$ simulations in order to observe the dynamics of the gating channels in the $\mathrm{HH}$ equations. The transient voltage field in the absence of mechanical deformation at $1 \mathrm{~ms}$ predicted by the 3D FE model with $\mathrm{HH}$ boundary conditions is shown in Figure B.11a. The comparison plot of the voltage profiles along the axon for is presented for all three codes in Figure B.11b.

A conservative element size was used here for the Neurite simulation, as an explicit scheme was used to generate the results. The time step of Neurite was also orders of magnitude smaller, however this was automatically determined by Neurite according to the element size and the scheme stability [28]. The 1D FE simulation required the smallest number of elements for spatial convergence, and a relatively large time step for temporal convergence. All simulations were temporally and spatially converged. The propagation profiles of CT in Figure B.10b and of $\mathrm{HH}$ in Figure B.11b are essentially 

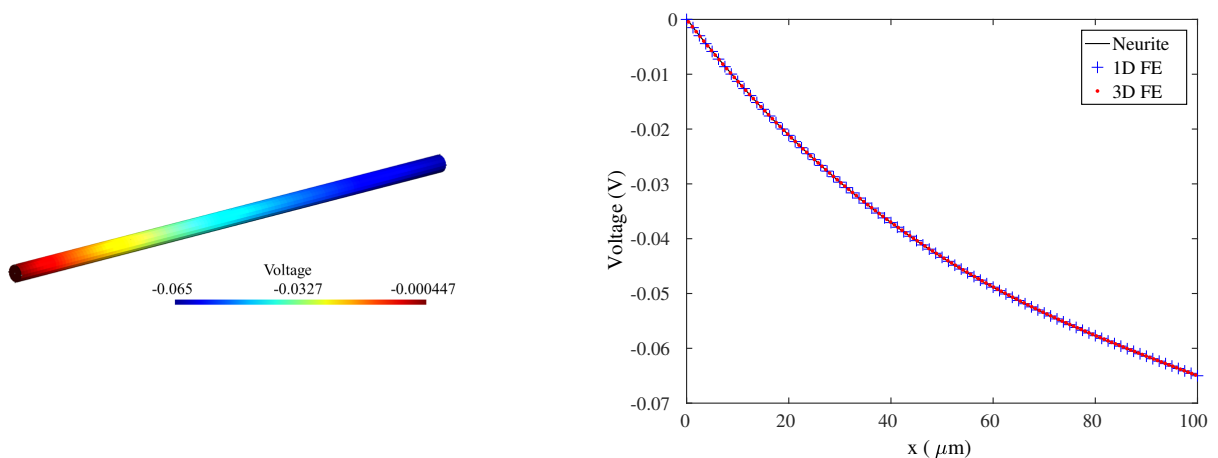

(a) Voltage profiles along the axon from 3D

(b) A comparison plot of simulations from FE simulations all three methods

Figure B.10: 3D FE internode simulation and comparison plot of propagation profiles along the axon between Neurite, 1D FE and 3D FE with CT boundary conditions at $50 \mu \mathrm{s}$.
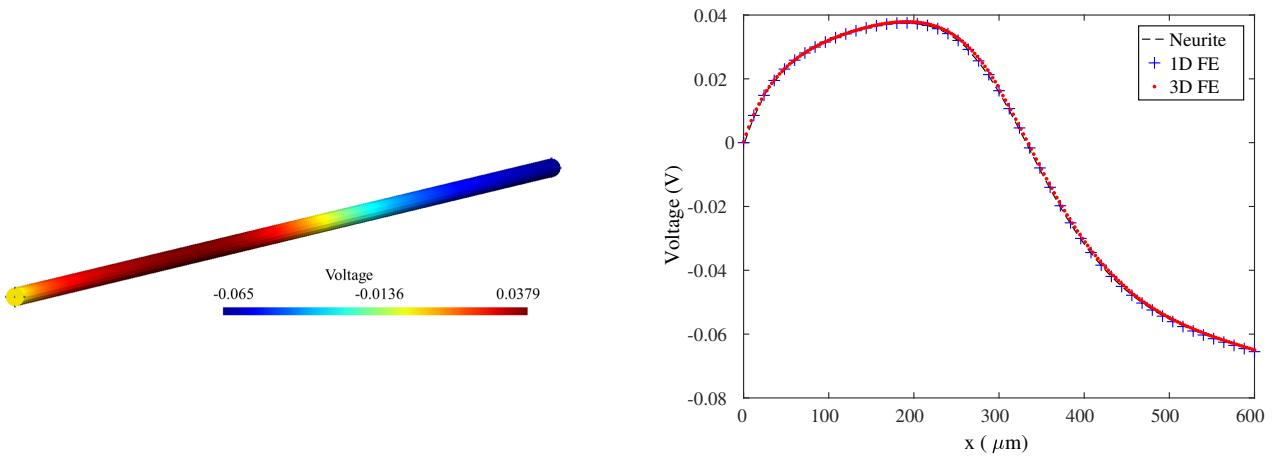

(a) Voltage profiles along the axon from 3D FE simulations

(b) A comparison plot of simulations from all three methods

Figure B.11: 3D FE node simulation and comparison plot of propagation profiles along the axon between Neurite, 1D FE and 3D FE with HH boundary conditions at $1 \mathrm{~ms}$. 
identical across all three methods, thus validating the implementation of the proposed scheme on a 3D FE platform.

\section{Appendix C. 1D FE script}

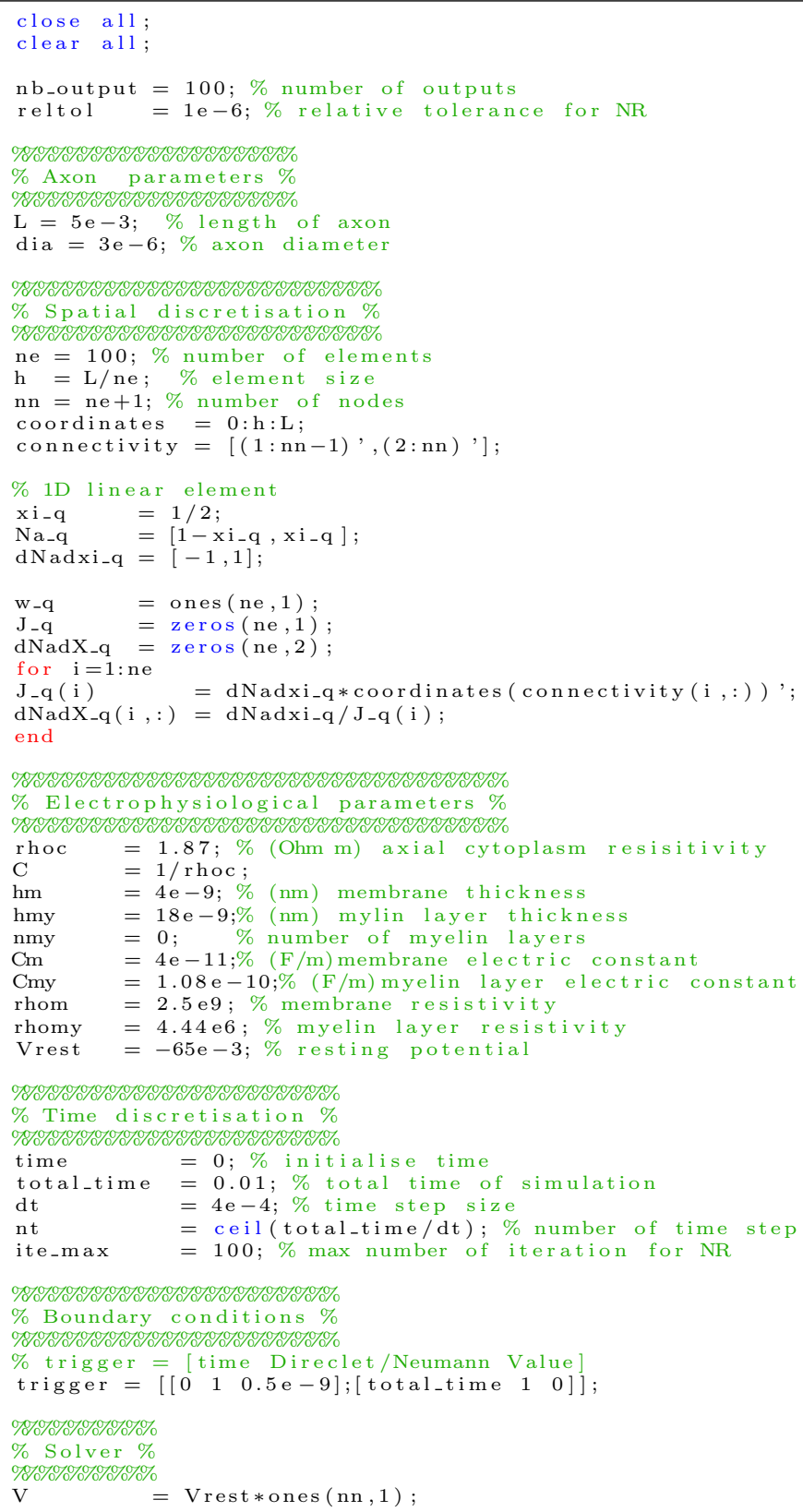




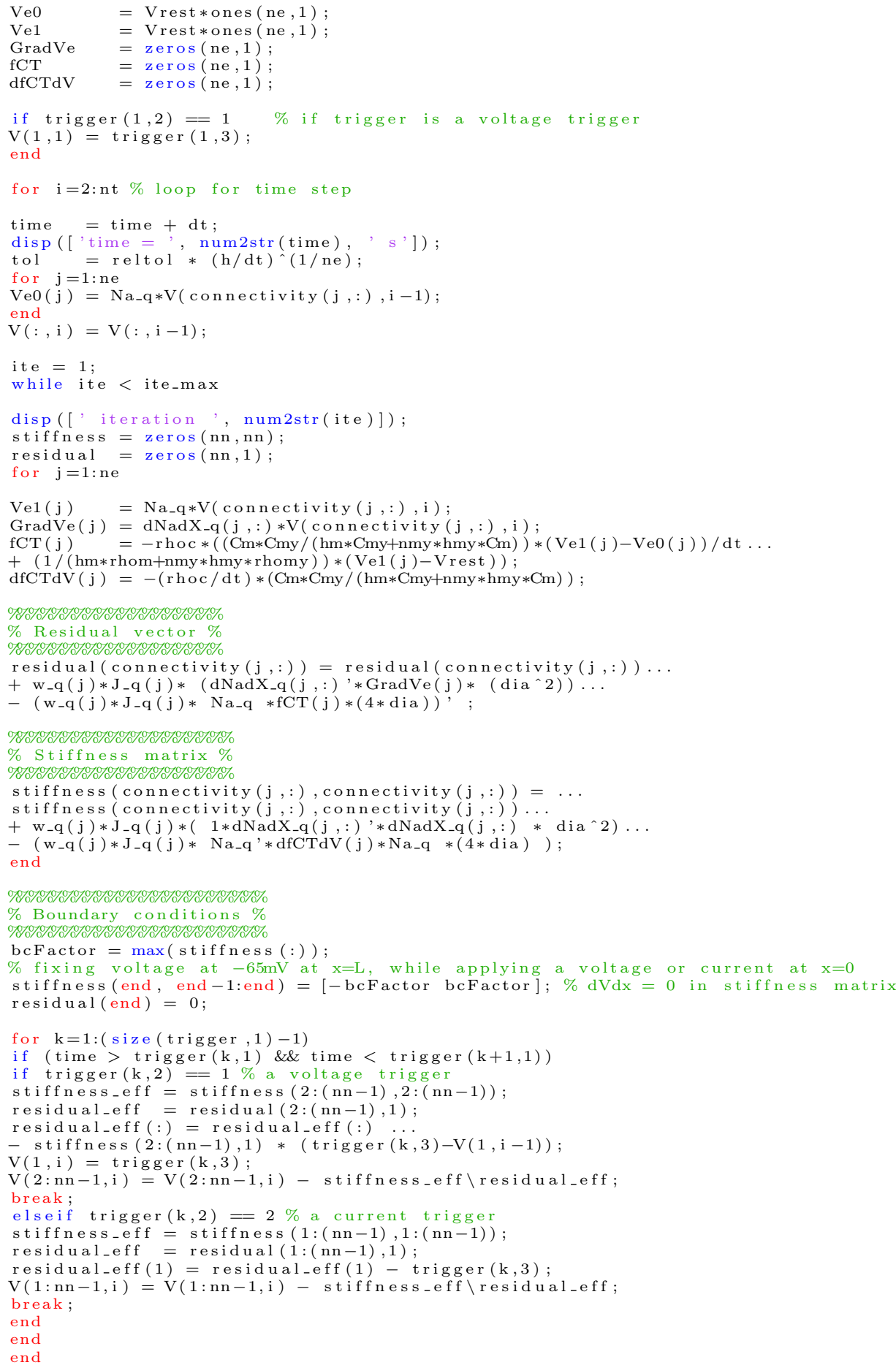




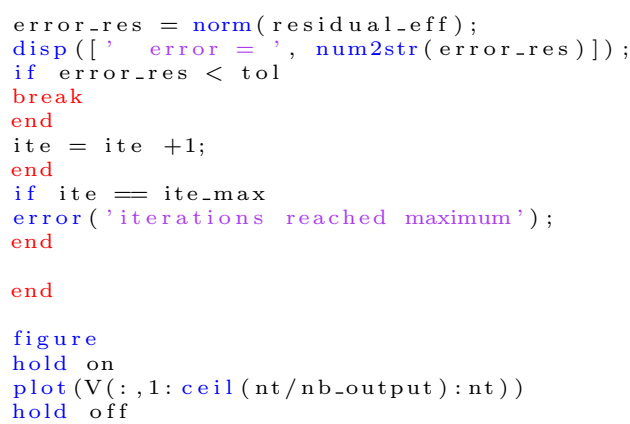

$\%$ This code is used to study HH electrophysiology with 1D FEM close all; 


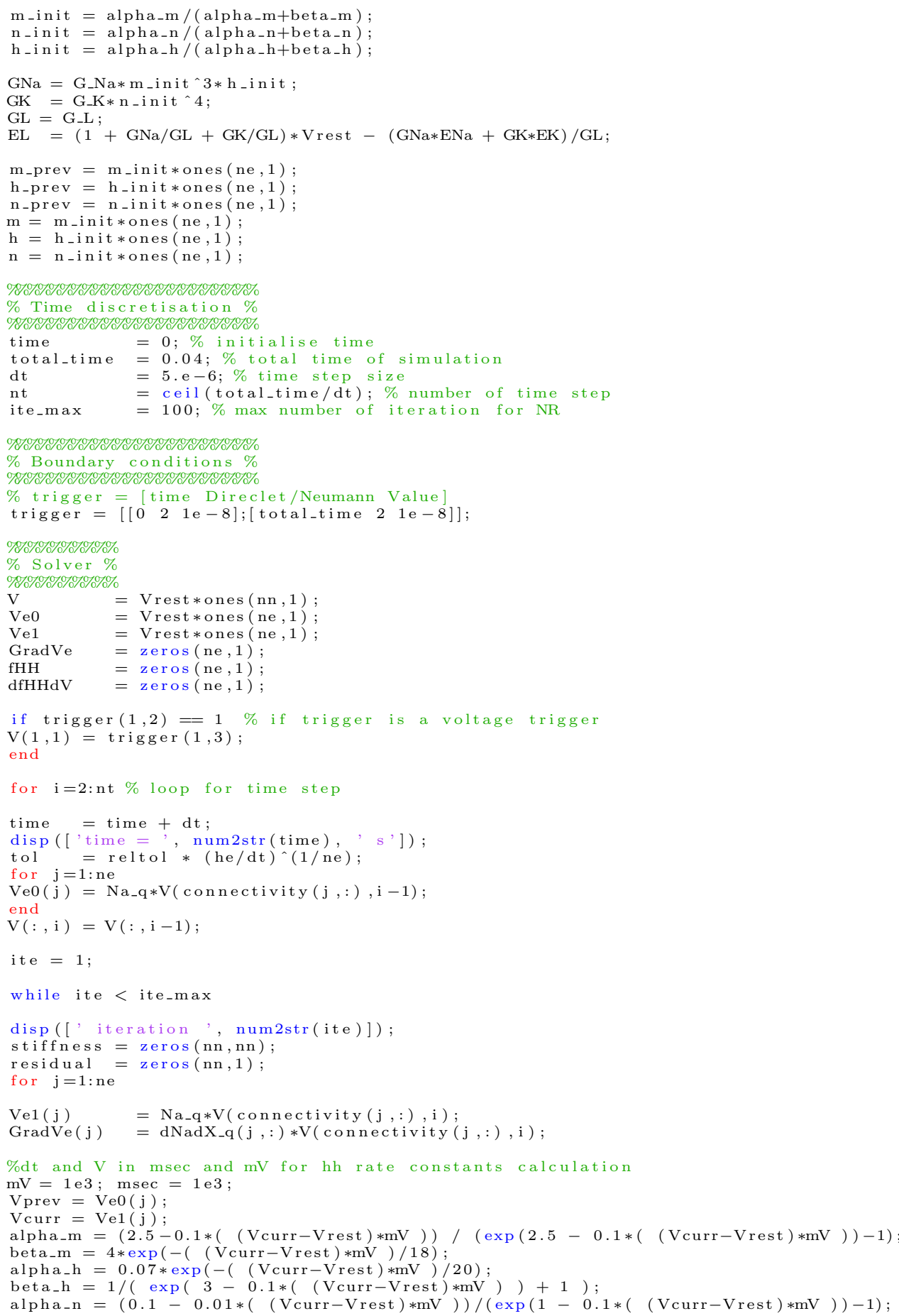




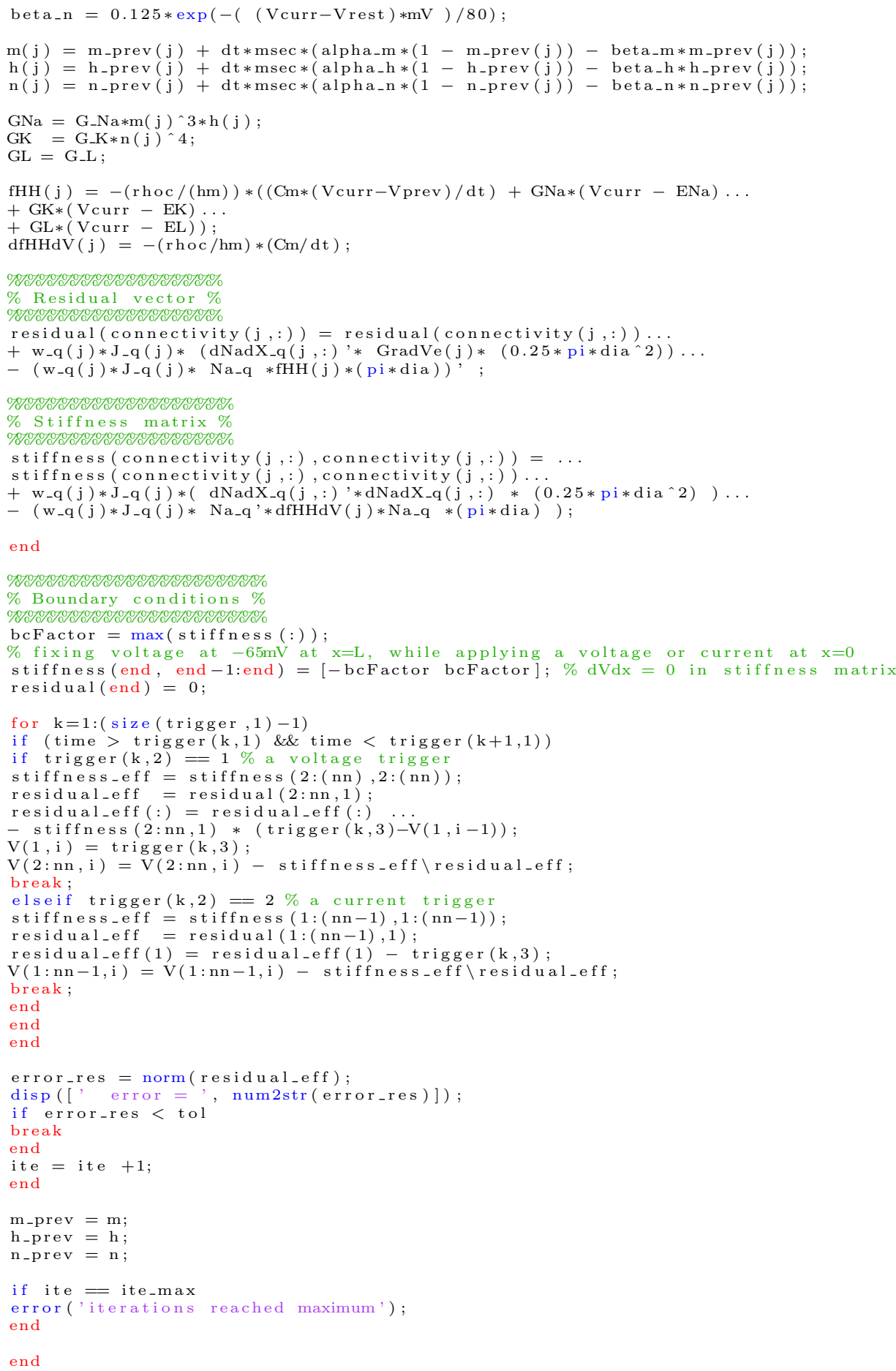


\begin{tabular}{l|l}
212 & \\
213 & figure \\
214 & hold on \\
215 & plot (coordinates $(:), V(:, 1:$ ceil $($ nt /nb_output $): n t))$ \\
216 & hold off
\end{tabular}

216 